\title{
Optimal control, MPC and MPC-like algorithms for wave energy systems: An overview
}

\author{
Nicolás Faedo*, Sébastien Olaya, John V. Ringwood \\ Centre for Ocean Energy Research, Maynooth University, Maynooth, Ireland
}

\section{A R T I C L E I N F O}

\section{Article history:}

Received 11 April 2017

Revised 20 June 2017

Accepted 2 July 2017

Available online 21 July 2017

\section{Keywords:}

Model predictive control

Receding horizon

Wave energy conversion

Wave energy device

Constrained optimisation

Optimal control

\begin{abstract}
A B S T R A C T
Model predictive control (MPC) has achieved considerable success in the process industries, with its ability to deal with linear and nonlinear models, while observing system constraints and considering future behaviour. Given these characteristics, against the backdrop of the energy maximising control problem for Wave Energy Converters (WECs), with physical constraints on system variables and a non-causal optimal control solution it is, perhaps, natural to consider the application of MPC to the WEC problem. However, the WEC energy maximisation problem requires a significant modification of the traditional MPC objective function, resulting in a potentially non-convex optimisation problem. A variety of MPC formulations for WECs have been proposed, with variations in the WEC model, discretisation method, objective function and optimisation algorithm employed. This paper attempts to provide a critical comparison of the various WEC MPC algorithms, while also presenting WEC MPC algorithms within the broader context of other WEC "optimal" control schemes.
\end{abstract}

(C) 2017 Elsevier Ltd. All rights reserved.

\section{Introduction}

Covering almost the $71 \%$ of the earth surface, the ocean is an almost inexhaustible source of clean energy. Energy capture from ocean waves has an enormous potential to fulfil the increasing worldwide energy demand, with an estimation of about 32,000 TWh/year (Mork, Barstow, Kabuth, \& Pontes, 2010). Nevertheless, wave energy is at an early stage of development (Edenhofer et al., 2011). Since the main difficulty with wave power is the irregular motion of the sea on different time scales (de O. Falcão, 2010), the technical and conceptual convergence to a device, best suited for this application, has not yet been achieved. As a result, hundreds of patents proposing different methodologies have been filed around the world (Pelc \& Fujita, 2002). An overview and classification of Wave Energy Converters (WECs) can be found in Drew, Plummer, and Sahinkaya (2009) and de O. Falcão (2010).

A WEC is a device used to harvest ocean wave energy, usually converting the mechanical energy of the waves to electrical energy through a Power Take-Off system (PTO). In order to be profitable, an optimised process, that ensures extracting the maximum time averaged power from ocean waves, is crucial. Moreover, such an optimisation strategy must take into account the physical limitations of the complete conversion chain in order to maximise power

\footnotetext{
* Corresponding author.

E-mail address: nicolas.faedo.2017@mumail.ie (N. Faedo).
}

absorption and minimise the risk of damage. Such an optimisation procedure can be achieved by designing an optimal controller that accomplishes this energy-maximising control objective.

Several optimal control formulations and methods have been developed in order to improve energy extraction from WECs, with extensive reviews available in studies such as Ringwood, Bacelli, and Fusco (2014a) and Ringwood, Bacelli, and Fusco (2014b). A brief discussion on the evolution of these kinds of controllers is also given in Section 3 .

A well-developed control strategy, within both the research and industrial control communities, that takes into account constraints, while optimising a given criterion, is Model Predictive Control (MPC). MPC was first suggested within the wave energy context in Gieske (2007). Subsequently, several studies began to implement different MPC strategies within the same context. Moreover, MPClike strategies, based on spectral and pseudospectral methods, have been developed and implemented in this field, in an attempt to overcome the computational burden of the original MPC strategies. Those MPC and MPC-like algorithms constitute the main scope of this study.

This review paper is organised as follows: First, the basics of WEC modelling, and physical constraints considered, are given in Section 2, while an overview of optimal control methods applied within wave energy conversion and, particularly, MPC strategies as an optimal formulation, can be found in Section 3. In order to provide a broader context, and a basis for comparison, brief summary on "traditional" MPC controllers can be found in Section 4. 


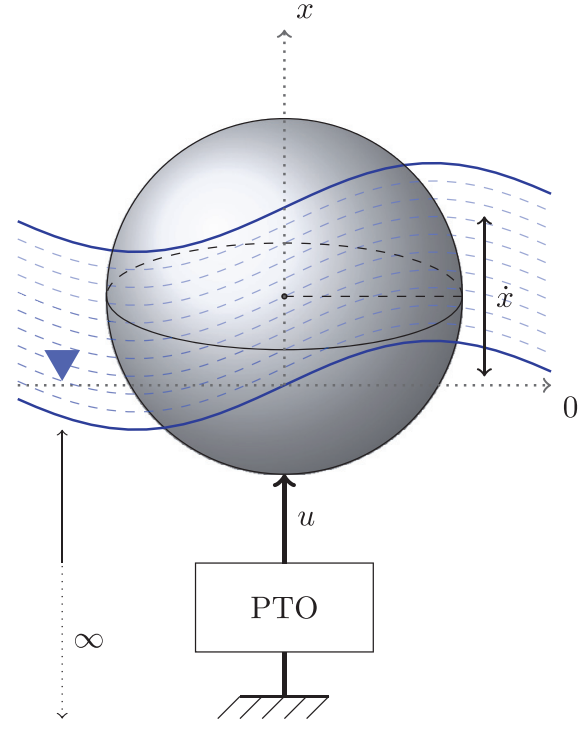

Fig. 1. Wave energy converter.

An extensive characterisation of MPC controllers in wave energy is given in Section 5, where the key elements of such strategies are analysed. Spectral and pseudospectral techniques (MPC-like algorithms) are presented in Section 6 and, finally, a discussion and further directions related to predictive control of WECs are given in Section 7.

\section{WECs: models and path constraints}

The WEC representation in Fig. 1 shows a spherical heaving point absorber, constrained to move in heave only, for simplicity. The device is assumed to be bottom referenced, with the opposing PTO force, $u(t)$, providing the control input. While the representation in Fig. 1 is for simple illustrative purposes only, a wide variety of WEC geometries and principles have been proposed (Koca et al., 2013).

In essence, the useful energy is converted in the PTO, calculated as the integral of converted power:

$E=-\int u(t) \dot{x}(t) d t$

where $\dot{x}(t)$ is the velocity of the device. The control problem can be informally described as the specification of $u(t)$ so that (1) is maximised. In general, if the resisting force $u(t)$ is larger, the velocity $\dot{x}(t)$ will be smaller, while a smaller resisting force will result in a greater velocity. Since the wave excitation force is bi-directional and multi-frequency, the determination of the optimal PTO force profile is non-trivial.

\subsection{WEC model}

In the majority of the published studies, the device is assumed to be a one degree-of-freedom system (1-DOF), constrained to move in heave only, such as that shown in Fig. 1. The WEC is referenced from its equilibrium position, immersed in an infinite-depth sea, in an undisturbed wave field. Such a system is then subject to fluid-structure interactions. These fluid-structure interactions are typically modelled using potential flow theory, where the fluid is assumed to be inviscid and incompressible, and flow is considered irrotational. Applying Newton's second law to the heaving body yields the linear hydrodynamic formulation:

$m \ddot{x}(t)=\mathcal{F}_{r}(t)+\mathcal{F}_{h}(t)+\mathcal{F}_{\text {exc }}(t)+u(t)$ where $m$ is the mass of the buoy, $x(t)$ the heave excursion, $\mathcal{F}_{\text {exc }}(t)$ the wave excitation force, $\mathcal{F}_{r}(t)$ the radiation force, $\mathcal{F}_{h}(t)$ the hydrostatic restoring force, and $u(t)$ is the control input applied through the PTO system. The hydrostatic force for a floating body is $\mathcal{F}_{h}(t)=\rho g V_{i}$ where $\rho$ is the water density, $g$ is the acceleration due to gravity, and $V_{i}=V_{0}-S x(t)$ represents the immersed volume of the WEC, with $V_{0}$ the immersed volume at the equilibrium position and $-S x(t)$ as an approximation of the additional immersed volume depending on the position of the WEC. The radiation force $\mathcal{F}_{r}$ is also modelled, based on linear potential theory and, using the well-known Cummins' equation (Cummins, 1962), is represented as

$\mathcal{F}_{r}(t)=-m_{\infty} \ddot{x}(t)-\int_{0}^{t} K(t-\tau) \dot{x}(\tau) d \tau$

where $m_{\infty}$ represents the added-mass at infinite frequency (further discussed in Section 3.1) and $K(t)$ is the (causal) radiation impulse response. Finally, the linearized equation of motion of the WEC is given as

$$
\left(m+m_{\infty}\right) \ddot{x}(t)+\int_{0}^{t} K(t-\tau) \dot{x}(\tau) d \tau+S_{h} x(t)=\mathcal{F}_{\text {exc }}(t)+u(t)
$$

where $S_{h}=\rho g S$ corresponds to the hydrostatic stiffness.

\subsection{Path constraints}

Real approaches to control solutions must consider the physical limitations constraining the body's motion and actuator characteristics. The importance of considering path constraints stems from the fact that the unconstrained solution that maximizes energy absorption (Falnes, 2002) is often impossible to achieve, due to excessive displacement (amplitude), velocity and/or force requirements. Other practical aspects related to the consideration of path constraints can be found, for example, in Genest, Bonnefoy, Clément, and Babarit (2014). Constraints considered in WEC control problems are often related to amplitude (position) $x(t)$, velocity $\dot{x}(t)$ and control input $u(t)$ which can be written (Genest \& Ringwood, 2016a) as,

$\forall t \in \mathbb{R},\left(X_{\max }, \dot{X}_{\max }, U_{\text {max }}\right) \in \mathbb{R}^{+^{3}},\left\{\begin{array}{l}|x(t)| \leq X_{\max } \\ |\dot{x}(t)| \leq \dot{X}_{\max } \\ |u(t)| \leq U_{\max }\end{array}\right.$

Constraints can be also found regarding the rate of change of the control input $\Delta u(t)$, and (unusually) the maximum power allowed by the PTO mechanism $P_{\max }$, as discussed in Section 5.2. A separate constraint relates to the direction of the power flow between the PTO system and the grid, termed a passivity constraint, according with the definition of passive systems: Consider a dynamical system with input $u(t)$ and output $y(t)$, where $y=h(t, u(t))$ and $t \in[0, \infty), u, y \in \mathbb{R}^{q}$; a system is said to be a passive system if $u(t) y(t) \geq 0, \forall t \in[0, \infty)$ (Khalil, 1996). The motivation to consider the analysis of unidirectional power flow is also discussed in Section 5.2, together with a description of studies reviewed that integrate this power flow constraint into their formulation.

\subsection{Summary of WEC modelling}

In summary, a wide variety of WEC devices with different operational principles can be found, since the conceptual convergence to a particular device has not been achieved yet, as discussed in Section 1 . The control objective for WECs is strongly (but not always exclusively) related to the useful energy absorbed (1), which directly depends on the velocity-force product for the PTO system. Despite the fact that the most common physical constraints considered for a WEC device are related to displacement, velocity and 


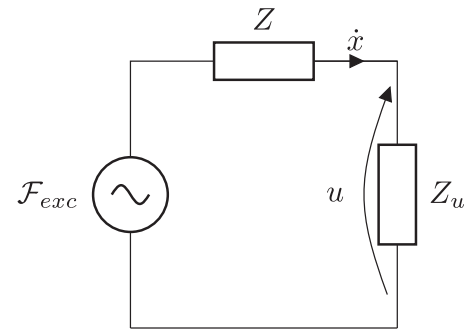

Fig. 2. Analogous electric circuit counterpart of the impedance matching problem for a WEC.

control input, additional constraints on the rate of change of the control force and even on the direction of the power flow between the device and the PTO system (passivity constraint) can also be important.

\section{Optimal control of WECs}

A general definition of the control problem for WECs can be given as follows (Ringwood etal., 2014b),

\section{Optimal control objective}

Maximize: Energy absorption

Subject to: Path constraints in (5)

A first approach to accomplish the energy-maximising control objective can be carried out by ignoring system constraints and considering a frequency-domain model with velocity $\dot{x}$ as output, and the sum of the excitation force $\mathcal{F}_{\text {exc }}$ and PTO force $u$ as input, obtained from (4) (Falnes, 2002) as

$$
\frac{\hat{\dot{x}}(\omega)}{\hat{\mathcal{F}}_{\text {exc }}(\omega)+\hat{u}(\omega)}=\frac{1}{Z_{i}(\omega)}
$$

with $\hat{\mathcal{W}} \equiv \mathscr{F}\{\mathcal{W}\}$, where $\mathscr{F}\{\bullet\}$ represents the Fourier transform of - and, particularly, $Z_{i}(\omega)$ represents the intrinsic impedance of the system (4), expressed (Falnes, 2002) as

$Z_{i}(\omega)=R_{r}(\omega)+j \omega[m+m(\omega)]+\frac{S_{h}}{j \omega}$

where $R_{r}(\omega)$ is the radiation resistance (which is real and even) and $m(\omega)$ is the frequency-dependent added mass. Assuming that $\hat{u}(\omega)=-Z_{u}(\omega) \hat{\dot{x}}(\omega)$, it is possible to derive the conditions for optimal energy absorption from (6), in the frequency-domain (Falnes, 2002), as

$Z_{u}^{o p t}(\omega)=Z_{i}^{*}(\omega)$

where the operator $\{\bullet\}^{*}$ denotes the complex conjugate of $\bullet \in$ $\mathbb{C}$. The choice defined in (8) is alternatively termed as complexconjugate (CC) control (Salter, 1979), reactive control (Budal \& Falnes, 1980) or impedance-matching control. The last term originates within electrical engineering, where the condition for maximum power transfer between an electrical generator and a complex load is analogous to (8), and is represented in Fig. 2. An alternative expression of (8) is given by the following two conditions (Falnes, 2002),

Amplitude: $\quad\left|\hat{\dot{x}}^{\text {opt }}(\omega)\right|=\frac{\left|\hat{\mathcal{F}}_{\text {exc }}(\omega)\right|}{2 R_{r}(\omega)}$

Phase: $\quad \cos (\phi)=1$

where $\phi$ is the phase difference between the phasors of the velocity $\dot{x}$ and the excitation force $\mathcal{F}_{\text {exc }}$. A control strategy based on satisfying both conditions in (9) is known in the literature as phase and amplitude (PA) control (Budal \& Falnes, 1977). A block diagram of both CC and PA control strategies is given in Fig. 3.

The conditions presented in both (8) and (9) have been developed by pursuing the maximisation of the average power absorbed

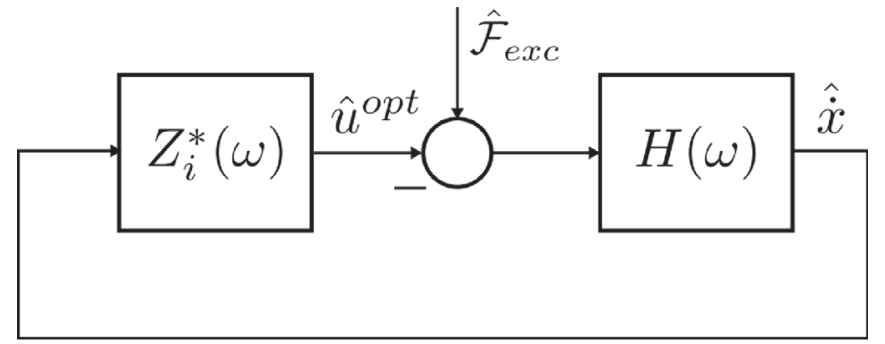

(a) Complex-Conjugate Control

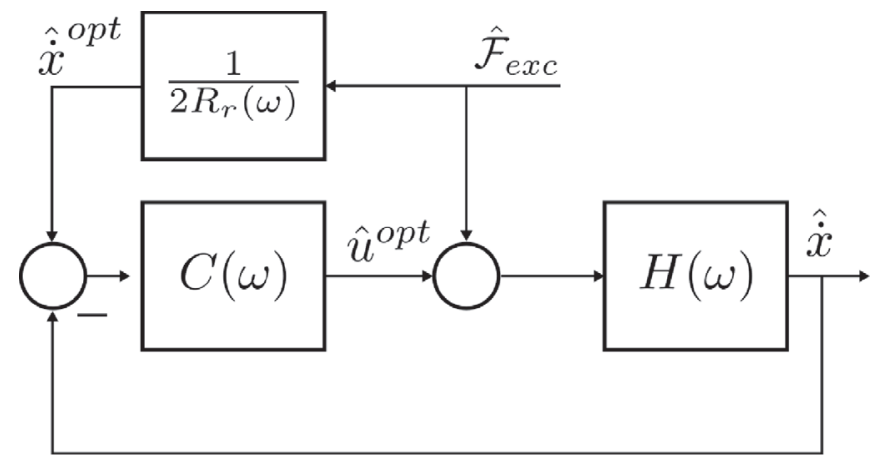

(b) Phase and Amplitude Control

Fig. 3. Block diagram of CC (a) and PA (b) optimal control strategies. $H(\omega)$ represents the input-output dynamics of the WEC using velocity as measurable variable. In (b), $C(\omega)$ is the transfer function of a controller designed for tracking the optimal velocity reference profile $\hat{\dot{x}}^{\text {ppt }}$.

by the WEC. Under the same assumptions, both control strategies lead to the same results in terms of motion, forces and converted power, differing in the method by which they achieve that objective (Falnes, 2002). However, the absorbed energy obtained by applying CC and PA control is achieved at the cost of large motions, large forces and power fluctuations. Some other important implications are listed in the following,

- Both results, namely (8) and (9), are frequency dependent; there is a different optimal control policy for each frequency, making its application difficult in irregular seas containing several frequencies.

- The control problem resulting from the optimality conditions of (8) and (9) is, in general, non-causal; due to $\mathscr{F}^{-1}\left\{Z_{i}^{*}\right\}$, which is non-causal, $\left.u^{o p t} \equiv u\right|_{Z_{u}=Z_{u}^{\text {opt }}}$ is dependent of future values of $\dot{x}$ in (8) or of $\mathcal{F}_{\text {exc }}$ in (9) (Falnes, 2002). This non-causal behaviour is further discussed in Section 3.1.

- Finally, (8) and (9) take no account of path constraints, making the optimal solution unrealistic.

Pioneering studies, regarding the optimal control of WECs, can be found in Evans (1979) and Falnes (1980), where the analytical formulations of the maximum power absorbed by a system of oscillating devices were developed independently. A review of the theory behind wave energy absorption can be found in Evans (1981b). A comprehensive description of optimal power absorption theory can be found in Falnes (2002), while an overview of the theory of optimal WEC control is given in Korde (2000), where frequency-domain and time-domain approaches are examined and discussed. One of the first applications of reactive control is described in Salter (1979). A study of the effect of irregular waves on CC control can be found, for example, in Tedeschi, Molinas, Carraro, and Mattavelli (2010) and Tedeschi, Carraro, Molinas, and Mattavelli (2011), where an analysis of instantaneous power is performed in addition to the consideration of average power. 


\subsection{Optimal non-causal control}

A brief discussion on the causality of the optimal control formulations in both $C C$ (8) and PA (9) controllers is given in the following, since such non-causal behaviour is a significant challenge associated with both $C C$ and $P A$ optimal controllers. Using the inverse Fourier transform, the impulse response $\mathscr{F}^{-1}\left\{Z_{i}(\omega)\right\}=z_{i}(t)$, associated with the mechanical impedance $Z_{i}(\omega)$, is non-causal (Falnes, 2002), since

$z_{i}(t)=K(t)+\left(m+m_{\infty}\right) \dot{\delta}(t)+\frac{1}{2} S_{h} \operatorname{sign}(t)$

where $\mathscr{F}^{-1}\left\{Z_{r}(\omega)-j \omega m_{\infty}\right\}=K(t)$ is the (causal) radiation impulse response as defined in Eq.(3), $\dot{\delta}(t)$ is the first generalized derivative of the Dirac delta function and $\operatorname{sign}(t)$ is the signum function, which is non-zero for $t<0$ if $S \neq 0$, and hence the impulse response $z_{i}(t)$ is non-causal. $m_{\infty}$ is the infinite-frequency added mass asymptote, i.e. $\lim _{\omega \rightarrow \infty} m(\omega)$. The intrinsic mechanical impedance can be modified to make the corresponding impulse response causal, by adding the term $S_{h} \pi \delta(\omega)$, as

$\tilde{Z}_{i}(\omega)=Z_{i}(\omega)+S_{h} \pi \delta(\omega)$

Then, using the inverse Fourier transform, $\tilde{z}_{i}(t)$ is evaluated as

$\tilde{z}_{i}(t)=K(t)+\left(m+m_{\infty}\right) \dot{\delta}(t)+S_{h} U(t)$

where $U(t)$ is the Heaviside unit step function. The impedances $Z_{i}(\omega)$ and $\tilde{Z}_{i}(\omega)$ differ only for $\omega=0$, which is not relevant in practice, because the mean velocity of oscillating WECs is generally zero (Bacelli, 2014). It follows that the CC controller, with an impedance defined as in Eq. (8), is non-causal: Let $\tilde{z}_{i}(t)$ be expressed (Falnes, 2002) as

$\tilde{z}_{i}(t)=\tilde{r}_{i}(t)+\tilde{x}_{i}(t)= \begin{cases}2 \tilde{r}_{i}(t) & \text { for } t>0 \\ \tilde{r}_{i}(0) & \text { for } t=0 \\ 0 & \text { for } t<0\end{cases}$

where the even part $\tilde{r}_{i}(t)$ and odd part $\tilde{x}_{i}(t)$ of $\tilde{z}_{i}(t)$, are the inverse Fourier transforms of $\operatorname{Re}\left\{\tilde{Z}_{i}(\omega)\right\}$ and $\operatorname{Im}\left\{\tilde{Z}_{i}(\omega)\right\}$ respectively. Then, the inverse Fourier transform of $\tilde{Z}_{i}^{*}(\omega)$ is

$\tilde{z}_{i}^{*}(t)=\tilde{r}_{i}(t)-\tilde{x}_{i}(t)=\tilde{r}_{i}(-t)+\tilde{x}_{i}(-t)=\tilde{z}_{i}(-t)$

Therefore, the impulse response associated with the CC controller is non-causal, if $\tilde{z}_{i}(t)$ is causal. In particular, it vanishes when $t>0$, due to the causality of $\tilde{z}_{i}(t)$. Also, the optimal PA controller calculation (9) is non-causal, because the transfer function $\frac{1}{R_{r}(\omega)}$ is real and even in $\omega$, and consequently, the associated impulse response is non-zero for $t<0$. This can be further appreciated in Fig. 4, where both the radiation impulse response $K(t)$ in $\tilde{z}_{i}(t)(12)$, and its counterpart in $\tilde{z}_{i}^{*}(t)(14)$ are shown. Results are computed for a spherical heaving point absorber WEC (as illustrated in Fig. 1), of $5 \mathrm{~m}$ radius. The non-causality of the CC controller (8) implies that the computation of the optimal control force $u^{\text {opt }}$ requires future knowledge of the velocity $\dot{x}$ of the device, while the noncausality of the PA formulation (9) implies that the calculation of the optimal velocity profile $\dot{x}^{\text {opt }}$ requires future knowledge of the excitation force $\mathcal{F}_{\text {exc }}$.

Since the optimality conditions developed in (8) and (9) cannot be achieved exactly (because of the required perfect prediction of either velocity or excitation force), sub-optimal causal controllers have been proposed. One of the most common approaches is to obtain a causal transfer function that approximates the ideal nonrealisable transfer function in the frequency interval containing most of the wave energy (Falnes, 2002). For example, in Clément and Maisondieu (1993), two causal approximations are considered for a simple device and compared with the ideal non-causal controller. Several other contemporary studies also consider the development of causal controllers. In Scruggs (2011) and Scruggs, Lattanzio, Taflanidis, and Cassidy (2013), a linear quadratic Gaussian

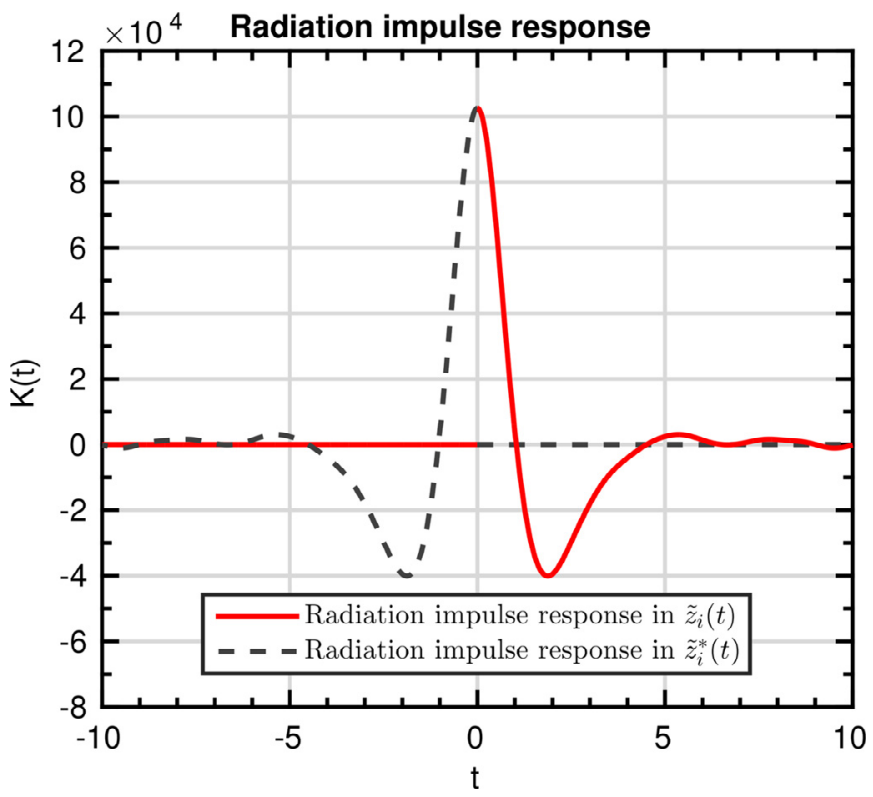

Fig. 4. Contribution of $K(t)$ in the impulse response of $\tilde{Z}_{i}(\omega)$ and $\tilde{Z}_{i}^{*}(\omega)$ for a heaving point absorber WEC of 5 metres of radius.

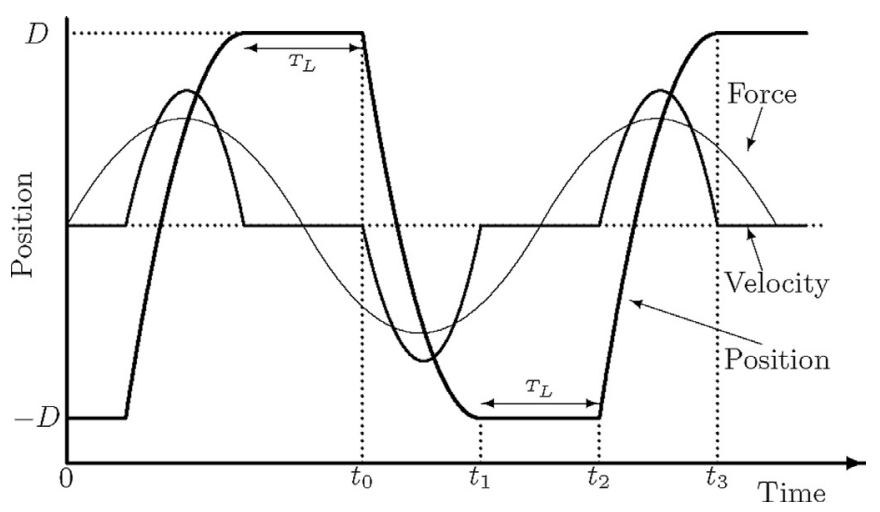

Fig. 5. System variables under latching control [7].

controller is designed for a 3-DOF WEC, subject to constraints; an extension of Scruggs etal. (2013) can be found in Nie etal. (2016), which can be used to accommodate nonlinearities in the system dynamics; causal stochastic optimal control is implemented in Nielsen, Zhou, Kramer, Basu, and Zhang (2013), based on the spectral characteristic of the wave elevation, which is optimal under monochromatic wave excitation, while a completely different causal approach is considered in Mundon, Murray, Hallam, and Patel (2005), where a phase controller is developed using genetic search techniques.

\subsection{Latching and de-clutching}

Inherently discrete sub-optimal controllers have been developed with different strategies using the conditions in (9). Several researchers (Budal \& Falnes, 1975; French, 1979; Guenther, Jones, \& Brown, 1979), proposed a control strategy termed latching which aims to satisfy the phase condition only (9b). The excitation force is kept in phase by locking the wave absorber in a fixed position for an appropriate time interval (Ringwood et al., 2014b). With latching, the control action is made at discrete instants of the cycle, as can be appreciated in Fig. 5: Latching is applied at time $t_{1}$, with the device released at $t_{2}$ after a latching duration $T_{L}$, with a similar protocol for positive displacements. Latching effectively 
increases the potential energy during each half cycle (Ringwood et al., 2014b).

One advantage of latching control is that any negative energy flow to the PTO is eliminated, easing the requirement of high conversion efficiency, when compared to the optimal formulation. Latching also deals with non-causality, specifically when computing the optimal latching interval (Naito \& Nakamura, 1986). However, a difficulty arises when the incident wave contains several frequency components and the concept of phase between excitation force and velocity is no longer well-defined, in which case the solution of optimising the latching interval is not unique (Babarit, Duclos, \& Clément, 2004). A comparison of several latching control strategies in regular (semi-analytical solution) and irregular (numerical solution) seas can be found in Babarit etal. (2004). In Babarit and Clément (2006), two methods to compute the optimal latching time for regular and random seas are considered. The first method is based on an analytical solution, while the second method is based on Pontryagin's maximum principle (Hoskin \& Nichols, 1987). A counterpoint to latching, termed de-clutching, is developed in Babarit, Guglielmi, and Clément (2009) and Babarit, Mouslim, Guglielmi, and Clément (2008). In de-clutching, the device is unloaded at specific time instants during the cycle. Studies on derivative-free optimisation have been performed for latching (Feng \& Kerrigan, 2013), de-clutching (Feng \& Kerrigan, 2014) and combined latching/de-clutching (Feng \& Kerrigan, 2015). A noteworthy observation has been made in Teillant, Gilloteaux, and Ringwood (2010) which states that (in general), for monochromatic seas and linear models, latching (de-clutching) is optimal when the device resonant period is shorter (larger) than the wave period.

\subsection{Constrained control}

Constraints must be considered as part of the optimisation problem, since the WECs are built using mechanical components which have limited operating ranges, as discussed previously in Section 2.2. An early theory for the maximisation of wave-power absorption of a system of oscillating bodies in the frequencydomain is presented in Evans (1981a) subject to amplitude restrictions, while a time-domain formulation for the energy-maximising objective can be found in Eidsmoen (1996a), also subject to amplitude constraints. In Eidsmoen (1996b); 1996c) and Eidsmoen (1998), phase control of a WEC subject to amplitude constraints is considered. The phase control applied produces results similar to latching, and aims to keep the velocity in phase with the excitation force $(9 b)$.

\subsection{Summary of optimal control of WECS}

In summary, the optimal control problem for WECs is noncausal. This either requires predictive control action (requiring wave forecasting) or a causal approximation. Both of these solutions involve an approximation error. Some simple, discrete, control formulations can be effective (latching, de-clutching), but do not, in general, observe the physical limitations on the system variables. A combination of these requirements motivated researchers to adapt the popular optimal MPC strategy for wave energy applications.

\section{Model predictive control}

MPC has its origins in late seventies, due to Richalet, Rault, Testud, and Papon (1976) and Richalet, Rault, Testud, and Papon (1978), presenting Model Predictive Heuristic Control (MPHC), and Cutler and Ramaker (1980) with Dynamic Matrix Control (DMC). The main difference between MPHC and DMC concerns the type of dynamic model used (impulse response in MPHC and step response in DMC). In 1987, Generalized Predictive Control (GPC) (Clarke, Mohtadi, \& Tuffs, 1987) appeared, based on the CARIMA input-output model. Further efforts to develop MPC within a state-space framework can be found in Li, Lim, and Fisher (1989). A significant amount of MPC development has subsequently taken place (see, for example, Camacho and Alba, 2013; Garcia, Prett, and Morari, 1989 and Maciejowski, 2002). Thus, the term MPC refers to a wide family of model-based control strategies, which obtains an optimal control input by considering an optimisation procedure (minimisation or maximisation) over a finite future time horizon. Unifying features of MPC in discrete time (Camacho \& Alba, 2013) are:

- A mathematical model of the process, to predict the output at future time instants (horizon $N$ ). Typically, a discrete-time model is used, so that the continuous-time problem must be discretised with a chosen technique.

- An objective function $\mathcal{J}$, in order to obtain an optimal control sequence within the optimisation window. Obtaining such an optimal control policy involves a constrained or unconstrained minimisation (or maximisation) procedure, which can be solved with a variety of optimisation algorithms.

- A receding strategy where, at each instant $k$, the finite horizon is displaced towards the future, and only the first control signal of the optimal sequence calculated is applied at each step.

These algorithms are also called Receding Horizon Predictive Control. In particular, if nonlinearities are present in the system model, the algorithm is said to be a Nonlinear MPC (NMPC). The advantages of MPC include the handling of multi-variable systems in a straightforward manner, and compensation for dead time can be dealt with in an intrinsic way. Nevertheless, the most important aspect is constraint handling, which remains conceptually simple, and can be systematically included during the design process.

The methodology of all controllers within the MPC family is defined by the following strategy (Camacho \& Alba, 2013): at each iteration of the algorithm $k$, the whole vector of future values of the control signal $\mathbf{u}(k)=\left[u(k \mid k) \cdots u\left(k+N_{u}-1 \mid k\right)\right]^{T}$ is calculated on-line. $u(k+p \mid k)$ denotes the value of the control input for the sampling instant $k+p$ calculated at iteration $k$, and $N_{u}$ is the control horizon. Alternatively, the vector of increments of the future values of the manipulated variable $\Delta \mathbf{u}(k)=\mathbf{u}(k)-\mathbf{u}(k-1)$ can be used. This vector is found by minimising an objective function (constrained or unconstrained) that usually takes into account the tracking errors over the prediction horizon $N$ and a penalty term that encourages the reduction of excessive changes in the manipulated variable. The typical quadratic MPC objective function takes the form

$$
\mathcal{J}(k)=\sum_{p=1}^{N}\left[y^{s p}(k+p \mid k)-\hat{y}(k+p \mid k)\right]^{2}+\lambda \sum_{p=0}^{N-1}[\Delta u(k+p \mid k)]^{2}
$$

where $\lambda$ is a weighting coefficient, $y^{s p}(k+p \mid k)$ is the set-point value for the sampling instant $k+p$ known at the instant $k$ and $\hat{y}(k+p \mid k)$ is the predicted value of the output obtained with the mathematical model at the sampling instant $k+p$, calculated at the current iteration $k$. The prediction of the outputs is performed consecutively for $p=1, \ldots, N$. Regarding the receding strategy, only the first element is applied to the process at sampling instant $k$, that is $u(k)=u(k \mid k)$ or $u(k)=\Delta u(k \mid k)+u(k-1)$. At the next iteration $(k+1)$, the output measurement is updated and the procedure is repeated. While MPC applied to WECs also involves a mathematical model, a receding horizon strategy, and can deal with system constraints, the objective function contrasts 


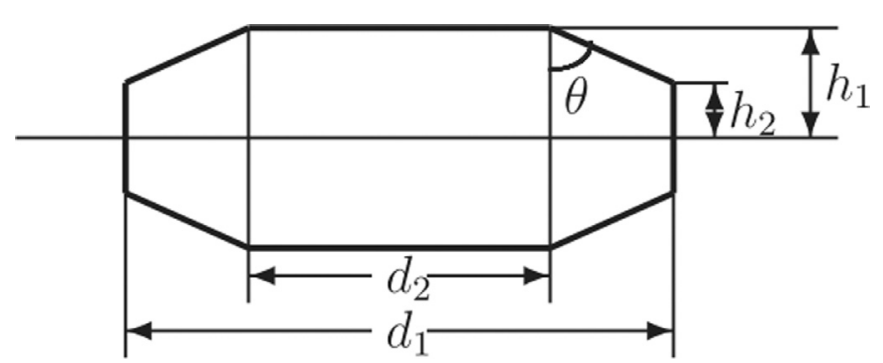

Fig. 6. The schematic diagram of the float with non-constant radius (Li, 2015).

significantly with the "tracking" objective of (15). Rather, a converted energy maximising objective, consistent with the definition of the WEC control problem at the start of Section 3 is employed, further detailed in Section 5.5. In particular, this variation can cause numerical search problems, due to the loss of convexity of the performance function (see Section 5.5). Some exceptions, where a traditional MPC objective is applied in the WEC field, are detailed in Section 5.

\section{Model predictive control in wave energy}

As stated in Section 4, the key distinguishing elements of MPC strategies relate to variations in dynamical models, objective functions and optimisation algorithms. Hence a categorisation of the reviewed literature in terms of models used (characteristics, representation and discretisation techniques), performance functions employed to accomplish the energy maximising objective, and optimisation algorithms implemented to solve such a problem, are given in the following subsections.

\subsection{WEC model}

A linear model structure similar to (4) is used in the vast majority of studies implementing MPC and MPC-like strategies in wave energy. However, differences can be observed in the literature concerning both MPC and MPC-like algorithms, where additional terms are sometimes included. Such relevant differences will be briefly detailed in the following. In Li, Weiss, Mueller, Townley, and Belmont (2012), a viscous force (termed a friction force) is considered, which can be written using an additional damping term, as in Falnes (2002). In Richter, Magaña, Sawodny, and Brekken (2014) a MPC strategy for a two-body WEC is proposed, with a linearised mooring-line term added to the model. In a subsequent study (Richter, Magaña, Sawodny, \& Brekken, 2013), the authors of Richter etal. (2014) develop a NMPC formulation for the same device, for the case where a nonlinear mooring-line term is considered. Such a mooring system is based on the configuration developed in Yu and Li (2011). Another variation can be found in Li and Belmont (2014a); 2014b) and Li (2015), where the convolution integral present in the equation of the radiation force (3) depends on the relative device/fluid velocity. An array of WECs is considered in Li and Belmont (2014b), where their interaction is derived from linear identification, based on experimental data. For numerical simulation, the authors use the fluid/mechanical model as described in Falnes (1980). Another interesting modification is presented in both Olaya, Bourgeot, and Benbouzid (2014) and Olaya, Bourgeot, and Benbouzid (2015), where the MPC strategy is applied to a two-body system using a one-body equivalent model, rather than a full two-body dynamic system.

A different formulation can be found in Tom and Yeung (2014), where a particular choice of the PTO system (permanent magnet generator), described as a time-varying damper, is studied. Amann, Magaña, and Sawodny (2015) also present a nonlinear mooring term, resulting in an NMPC formulation, which is based on the same mooring configuration presented in Richter etal. (2013). In Fig. 8, the nonlinear behaviour of such terms can be appreciated, which is also compared with a corresponding linear approximation. An alternative approach can be found in Kovaltchouk etal. (2015), where an energetic model of the buoy (using the fact that the model is linear and passive) is employed. In Li (2015), a further NMPC strategy is developed, where the model is the same as in Li and Belmont (2014a); 2014b) or Li et al. (2012), but includes a nonlinear buoyancy force, due to consideration of the non-uniform cross-sectional area of the device. Such a device is shown in Fig. 6.

An array of three WECs is considered in Oetinger, Magaña, and Sawodny (2014), where each device behaves like a heaving point absorber moored to the sea-floor, with a certain angle with respect to the surface, as in Vicente, Antonio, Gato, and Justino (2009). Finally, a different type of point absorber is considered in Andersen, Pedersen, Nielsen, and Vidal (2015) and Nguyen, Sabiron, Tona, Kramer, and Sanchez (2016), which is based on a real prototype sited in Hansen and Kramer (2011), composed of a number of hemispherically shaped floats attached to a single platform.

Other differences can be found in studies applying spectral and pseudospectral methods (see Section 6). In Bacelli, Ringwood, and Gilloteaux (2011) a self-reacting point absorber composed of two concentric asymmetric bodies is considered, while different WECs are used in Bacelli and Ringwood (2014) and Genest and Ringwood (2017) (flap-type device de O.Falcão (2010)), and Paparella and Ringwood (2017a) and Paparella and Ringwood (2017b) (multibody hinge-barge device Paparella, Bacelli, Paulmeno, Mouring, \& Ringwood, 2016). This last device is presented in Fig. 7, as an example of a multibody structure, among the different types of WECs.

In summary, a wide variety of WEC models are employed by various researchers, with some variation due to the disparity between WEC types, but also related to the limitations of the model complexity that can be tolerated by WEC MPC formulations with achievable solutions. The use of spectral methods is also responsible for some of the model variations. Overall, most MPC WEC

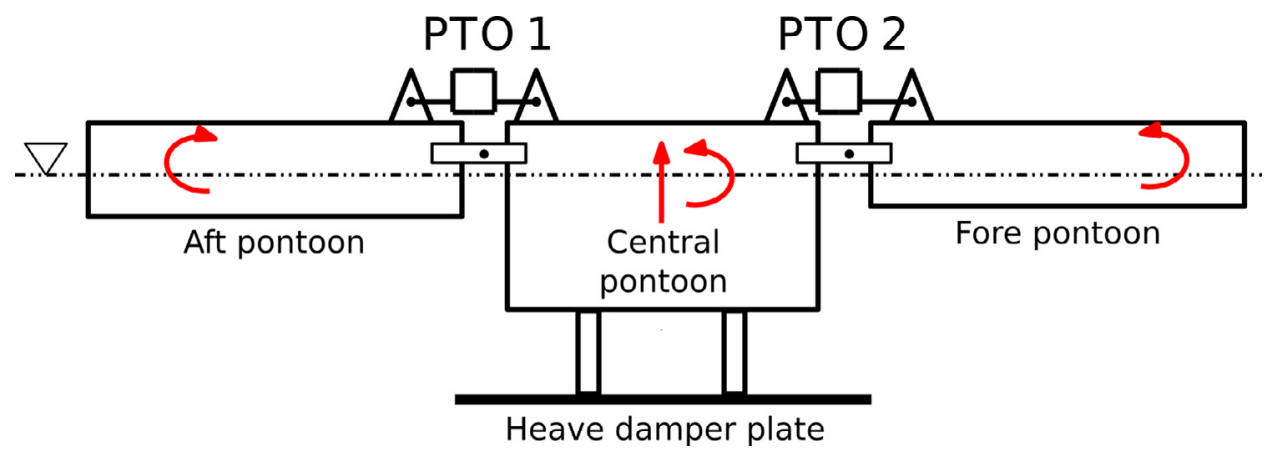

Fig. 7. Schematic diagram of a multibody hinge-barge device, as an example of a multibody WEC. Adapted from Paparella and Ringwood (2017a). 
Table 1

Considered path constraints.

\begin{tabular}{|c|c|}
\hline Variable & References \\
\hline$x(t)$ & $\begin{array}{l}\text { Abdelkhalik etal. (2016); Amann etal. (2015); Bacelli et al. (2013, 2015); Bacelli and Ringwood (2013); 2015); Bacelli etal. (2011); Brekken (2011); } \\
\text { Cavaglieri et al. (2015); Cretel et al. (2010); Cretel et al. (2011); de la Villa Jaén et al. (2014); Garcia-Rosa et al. (2015a); Genest and Ringwood (2016a); } \\
\text { 2016b); 2017); Hals etal. (2011); Herber and Allison (2013); Jama et al. (2014); Li (2015); Li and Belmont (2014a); 2014b); Li et al. (2012); Oetinger et al. } \\
\text { (2014); Olaya etal. (2014); O’Sullivan and Lightbody (2017); Richter etal. (2013); Richter et al. (2014); Tom and Yeung (2014); Westphalen et al. (2011) }\end{array}$ \\
\hline$\dot{x}(t)$ & $\begin{array}{l}\text { Amann et al. (2015); Bacelli et al. (2015); Brekken (2011); Cavaglieri et al. (2015); Cretel et al. (2011); Genest and Ringwood (2016a); 2016b); 2017); } \\
\text { Oetinger et al. (2014); Olaya et al. (2014); O'Sullivan and Lightbody (2017); Richter et al. (2013, 2014) }\end{array}$ \\
\hline$u(t)$ & $\begin{array}{l}\text { Abdelkhalik et al. (2016); Abraham and Kerrigan (2013); Amann et al. (2015); Andersen etal. (2015); Bacelli et al. (2013, 2015); Bacelli and Ringwood } \\
\text { (2013); 2015); Cavaglieri et al. (2015); de la Villa Jaén et al. (2014); Garcia-Rosa et al. (2015a); Genest and Ringwood (2016a); 2016b); 2017); Hals et al. } \\
\text { (2011); Herber and Allison (2013); Jama et al. (2013); Kovaltchouk et al. (2015); Li (2015); Li and Belmont (2014a); 2014b); 2014b); Li et al. (2012); } \\
\text { Nguyen etal. (2016); Oetinger etal. (2014); Olaya etal. (2014); 2015); O’Sullivan and Lightbody (2017); Richter etal. (2013); Richter et al. (2014); Soltani, } \\
\text { Sichani, and Mirzaei (2014); Tom and Yeung (2014); Tona et al. (2015) }\end{array}$ \\
\hline$\Delta u(t)$ & Brekken (2011); Li and Belmont (2014a). (Jama etal., 2014; Li \& Belmont, 2014b) \\
\hline$P_{\max }$ & Bacelli et al. (2015); Kovaltchouk et al. (2015) \\
\hline
\end{tabular}

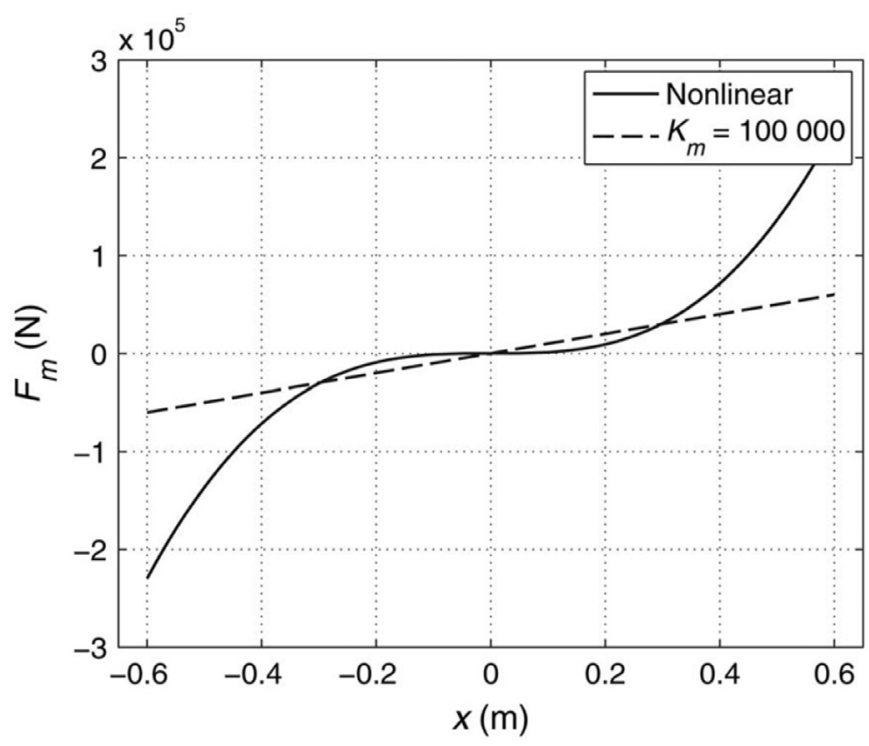

Fig. 8. Nonlinear eight-cable mooring force compared to the linearised mooring force with $K_{m}:=10^{5} \frac{\mathrm{N}}{\mathrm{m}}$ (Amann et al., 2015).

formulations utilise linear models, which can have dubious validity under an energy (motion) maximising philosophy (Giorgi, Retes, \& Ringwood, 2016).

\subsection{Path constraints}

As mentioned in Section 4, one of the main advantages of MPC algorithms is their ability to handle naturally constraints in the optimisation process. A comprehensive summary of constraints, considered in publications on both MPC and MPC-like algorithms for WEC control, can be found in Table 1. Most of the studies consider both amplitude (displacement) and control input force constraints, while other studies consider velocity constraints as well. Constraints on the rate of change of the control input are less common in the literature. It is important to mention that, in the case of Brekken (2011) and Jama, Wahyudie, Assi, and Noura (2014), the MPC algorithm is applied to a tracking aspect of WEC control, so that the model is augmented and the variable used in the optimisation process is, in fact, $\Delta u(t)$ (Camacho \& Alba, 2013). A further comment is needed with respect to Kovaltchouk et al. (2015), where a MPC strategy, that takes into account the main limitations of an electrical PTO chain (maximum force and maximum power) and possible losses, is proposed, and considers a constraint on the maximum absolute value of power $P_{\max }$, due to an intrinsic limitation of the proposed active rectifier. Another noteworthy difference can be found in Herber and Allison (2013), where an asymmetric control input constraint is considered, increasing the difficulty of implementation from an optimisation perspective. In addition, a variation arises in O'Sullivan and Lightbody (2017), where (besides constraints in position, velocity and the control input) authors consider nonlinear constraints due to voltage limitations in the power converters used to control the linear permanent magnet generator (Polinder, Damen, \& Gardner, 2004) (the PTO system), in order to avoid unreachable PTO forces and therefore, infeasible optimal solutions.

Reactive control (i.e. where bidirectional power flow between the PTO system and the grid is present), maximises energy absorption, as discussed in Section 3. However, this strategy comes with significant drawbacks, such as non-causality (as expressed in Section 3.1). Furthermore, this controller involves a large reactive power flux, as reported in, for example, Hals, Bjarte-Larsson, and Falnes (2002), where the maximum reactive power flow is shown to be ten times the average power. This presents a difficulty when implementing a reactive control policy using non-ideal actuators, since energy losses may be equivalent, or even larger, than the energy gained with the control strategy (Genest et al., 2014). Moreover, in Genest et al. (2014), the PTO efficiency is shown to be critical to power absorption; reactive control increases the power performance only if the actuator efficiency is greater than $80 \%$, which requires high efficiency components. These existing drawbacks, in the reactive control scheme, motivated researchers to study the optimal control formulation subject to constraints on the power flow direction, i.e. passivity constraints (as defined in Section 2.2). In the literature reviewed, studies that consider passivity constraints include, for example, Herber and Allison (2013); O'Sullivan and Lightbody (2017); Paparella and Ringwood (2017a) and Paparella and Ringwood (2017b).

In summary, constraints on displacement, velocity and force are frequently considered in the literature, as shown in Table 1 , though the issues of passivity (to prohibit reactive power flow) and peak power limitation (observing the rated power of the PTO) are far less common, but no less important. However, limitations on passivity or peak power make the numerical search problem significantly more challenging (Herber \& Allison, 2013) which, at least, partially explains their lack of treatment in the literature.

\subsection{Model representation: continuous-time state-space}

A MPC strategy can make use of various models to represent the relationship between the outputs and the measurable inputs (i.e. the system dynamics), as stated in Section 4. In MPC formulations, related to wave energy conversion, the most common representation is the state-space model. Several methods to obtain a continuous-time state-space representation from Cummins' equation (4) can be employed. The convolution product present in 
the radiation force (3) can be approximated with different methods, as described (for example) in Kristiansen, Hjulstad, and Egeland (2005); Pérez and Fossen (2008) and Roessling and Ringwood (2015). These strategies provide a particular state-space model for the approximated radiation force, given by,

$$
\dot{x}_{r}(t)=A_{r} x_{r}(t)+B_{r} \dot{x}(t)
$$

$\int_{0}^{t} K(t-\tau) \dot{x}(\tau) d \tau \approx C_{r} x_{r}(t)$

where $x_{r} \in \mathcal{X}_{r}$, such that the dimension of $\mathcal{X}_{r}$ is $n_{r}$ and consequently $A_{r} \in \mathbb{R}^{n_{r} \times n_{r}}, B_{r} \in \mathbb{R}^{n_{r} \times 1}, C_{r} \in \mathbb{R}^{1 \times n_{r}}$. Particularly, $n_{r}$ is the order of the finite-order approximation. As stated in Cretel, Lightbody, Thomas, and Lewis (2011), the components of $x_{r}(t)$ do not have any physical meaning, but still hold information about the state of the surrounding fluid. This subsystem, for the radiation force, which has the velocity of the device $\dot{x}(t)$ as input and radiation force as output, can now be included as a part of the overall model describing the motion of the device, developing a complete state-space model for the equation of motion (4) expressed as,

$\dot{x}_{M}(t)=A_{M} x_{M}(t)+B_{M}\left({ }^{n} \mathcal{F}_{\text {exc }}(t)+{ }^{n} u(t)\right)$

$y_{M}(t)=C_{M} x_{M}(t)$

where $x_{M}=\left[x, \dot{x}, x_{r}^{T}\right]^{T} \in \mathbb{R}^{\left(2+n_{r}\right) \times 1}$ is the state-vector of the continuous time model; $y_{M}=[x, \dot{x}]^{T} \in \mathbb{R}^{2 \times 1}$ represents the output vector of the system (assuming that position and velocity are both measurable outputs); and ${ }^{n} \mathcal{F}_{\text {exc }}(t),{ }^{n} u(t)$ corresponds to the excitation force and control input, both normalised by the total mass of the system, i.e. $m_{M}=m+m_{\infty}$. The matrices associated with the system (17) are given as follows,

$\begin{aligned} A_{M} & =\left[\begin{array}{ccc}0 & 1 & \mathbb{O}_{1 \times n_{r}} \\ -\frac{S_{h}}{m_{M}} & 0 & -C_{r} \\ \mathbb{O}_{n_{r} \times 1} & \frac{B_{r}}{m_{M}} & A_{r}\end{array}\right] B_{M}=\left[\begin{array}{c}0 \\ 1 \\ \mathbb{O}_{n_{r} \times 1}\end{array}\right] \\ C_{M} & =\left[\begin{array}{lll}1 & 0 & \mathbb{O}_{1 \times n_{r}} \\ 0 & 1 & \mathbb{O}_{1 \times n_{r}}\end{array}\right]\end{aligned}$

where $A_{M} \in \mathbb{R}^{\left(2+n_{r}\right) \times\left(2+n_{r}\right)}, B_{M} \in \mathbb{R}^{\left(2+n_{r}\right) \times 1}, C_{M} \in \mathbb{R}^{2 \times\left(2+n_{r}\right)}$ and $\mathbb{O}_{a \times b}$ represents a null matrix of size $(a \times b)$. Finally, the state-space model of (17) is represented by $\left(2+n_{r}\right)$ variables for the dynamic description. The dimension of the complete state-space increases with the dimension of $\mathcal{X}_{r}$, which results in an important trade-off in the optimisation procedure; choosing a higher (and hence more accurate) radiation force description results in a higher computational burden for the MPC strategy, which can lead to computational requirements that preclude real-time application. The accuracy of radiation force approximations, regarding the order $n_{r}$, is discussed in Tom and Yeung (2014). Simulations from same study are presented here, in Fig. 9, where the error between the radiation kernel function calculated with the inverse Fourier transform (IFT) (as in Cummins (1962)), and the approximated state-space model, is presented, for various values of $n_{r}$.

Most of the studies (that actually specify $n_{r}$ in an explicit way) consider an approximation order between 3 and 6 , such as Abraham and Kerrigan (2013); Cretel, Lewis, Lightbody, and Thomas (2010); Cretel etal. (2011); Hals, Falnes, and Moan (2011); Jama, Wahyudie, Assi, and Noura (2013); 2014); Kovaltchouk etal. (2015); Li (2015); Tom and Yeung (2014); Tona, Nguyen, Sabiron, and Creff (2015) and de la Villa Jaén, Santana etal. (2014). Exceptions beyond $n_{r} \in\{3,4,5,6\}$ are also possible, thought the choice of $n_{r}$ is not necessarily straightforward, since the modelling error is not always unimodel in $n_{r}$. Some studies consider the radiation convolution approximation (16) as a damping term, such as $\mathrm{Li}$ and Belmont (2014a); Richter etal. (2014) and Brekken (2011). Another case is given in Li etal. (2012), where the radiation force is not considered in the development of the state-space model, although

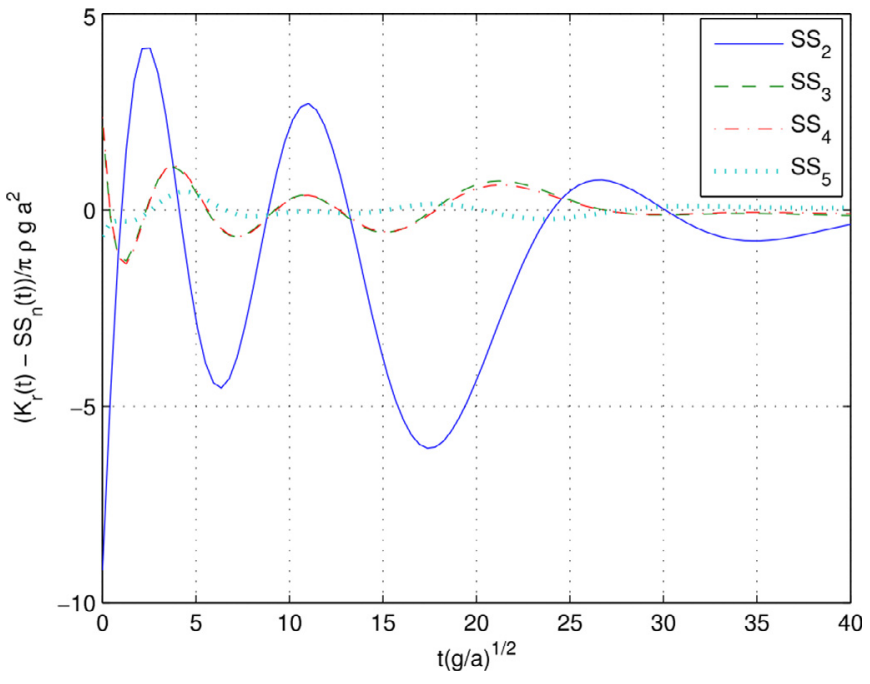

Fig. 9. Error between the radiation impulse response $K(t)$ (in fig as $K_{r}(t)$ ) calculated with IFT and different order state-space model approximations (in figure as $S S_{n_{r}}$ ). The factor $\pi \rho g a^{2}$ in the denominator of the error is used to provide dimensionless values (Tom \& Yeung, 2014).

that changed in later studies, namely Li and Belmont (2014a); 2014b) and Li (2015). In Li and Belmont (2014b), three MPC strategies for an array of WECs are described, which will be further discussed in Section 5.5. In Richter et al. (2013), two different nonlinear WEC models are considered: an extended model, including an approximation of the radiation convolution product, which is used for plant simulation only, and a reduced model, where authors assume constant inertia and additional linear viscous damping terms, claiming that convolution terms in radiation forces are "fairly small compared to other external forces". However, the authors of Richter etal. (2013) indicate that, if required, a full WEC model could be used in the MPC formulation, based on a linear state-space approximation as in (17) (the reader is referred to Richter (2011) for further details). Another noteworthy study is Amann et al. (2015), where a nonlinear mooring model, as described in Section 5.1, is considered. The authors claim that "the radiation forces, [...], are negligibly small and can be excluded for the given device". However, they also claim that, if necessary, a linear radiation approximation can be included, without changing the model structure.

In summary, several state-space representations for the equation of motion (4) are considered in the literature, by using an approximation of the radiation force, as in (16). The main difference between studies relates to the order $n_{r}$ of the approximation, which impacts directly on the dimension of the state-space model involved in the optimisation procedure. This results in a trade-off between the accuracy of the WEC model utilised in the design of the controller, and the real-time capabilities of the MPC formulation.

\subsection{State-space model discretisation}

In order to develop and implement a MPC strategy, the classical MPC approach uses a discretised version of the continuoustime state-space model, given in (17), to obtain the vector of the future outputs over the desired finite-time horizon. Such a discretisation can be achieved in several ways, with different results. The most common procedure (and the most popular among the reviewed studies) is the standard Zero-Order Hold ( $\mathrm{ZOH})$ method. Thus, references of studies that implement alternative discretisation techniques (apart from $\mathrm{ZOH}$ ) are highlighted in this section. The Triangular Hold, also known as a First-Order Hold (FOH) (Franklin, Powell, \& Workman, 1998), is used in Cretel et al. (2011); 
de la Villa Jaén etal. (2014) and Genest and Ringwood (2016a). In Cretel etal. (2011), the authors use a FOH, claiming that such a discretisation (combined with a modification of the objective function, explained in Section 5.5) "yields better results than those reported earlier". Such steps were also followed by dela Villa Jaén et al. (2014); Genest and Ringwood (2016a) (for comparative purposes) and O'Sullivan and Lightbody (2017). A Second-Order Hold is used in Richter et al. (2013), claiming that a discretisation of order 1 is not appropriate for the proposed NMPC approach. Another case is presented in Tom and Yeung (2014) and Kovaltchouk etal. (2015), where a trapezoidal rule is used. In Tona etal. (2015), the system dynamics are discretised using Tustin's method. One must recognise that such a discretisation results in a system representation which is not strictly proper, i.e. a non-zero feed-through matrix appears in (17). Another alternative discretisation method is used in Amann etal. (2015), where a second-order truncated Taylor series around the current time step is used, since the authors in Amann etal. (2015) report that the nonlinearities present in their model are not well approximated with the first derivative. A similar approach is also used in Richter (2011). In Jama et al. (2014), only the control input is expanded with a basis of Laguerre functions (Wang, 2001), which has a certain degree of similarity with the characteristic orthonormal expansion used in spectral and pseudospectral methods, discussed in Section 6.

In summary, despite the fact that the most common discretisation approach is the $\mathrm{ZOH}$, different (and diverse) discretisation methods can be found in the literature reviewed. The choice of technique to discretise the model mainly relates to the intrinsic dynamics considered (with possible nonlinearities). Each discretisation technique captures the dynamic of the system differently, affecting both the performance of the controller and the computational burden involved in the optimisation procedure. Nevertheless, all the discretisation techniques considered among MPC formulations in wave energy, can be considered as relatively "standard", in the sense that are well-known within the control community. In contrast, as discussed in Section 6, spectral and pseudospectral techniques, developed in the wave energy context, use a problemspecific parameterisation of the solution, discretising the dynamics of the system in a potentially more efficient way and, therefore, diminishing the computational effort required for the recedinghorizon control formulation.

\subsection{Objective function}

Considering the objective function, MPC, applied to wave energy conversion, deviates significantly from traditional MPC, as detailed in Section 4. In the WEC case, the objective function is strictly related to energy absorption, instead of the classical problem of reference tracking (15). The main objective of a wave energy device is to harvest energy from the incoming wave field in which the device is immersed. A control force $u(t)$, as the loading, is applied by the means of the PTO system. Thus, the control objective is to maximise the absorbed energy $E$ over a certain prediction horizon $T_{h}$, where

$E \equiv \mathcal{J}=-\int_{t}^{t+T_{h}} u(\tau) \dot{x}(\tau) d \tau$ while respecting the path constraints defined in (5). In this way, the general optimal control objective can be formulated as,

$\min _{u, \dot{x} \in \mathcal{C}\left[t, t+T_{h}\right]}-\mathcal{J}+\mathcal{J}^{\star}$

subject to, for $t \in\left[t, t+T_{h}\right]$ :

System dynamics given by (4) or (17)

Path constraints given by (5)

where $\mathcal{J}^{\star}$ represents additional terms considered in the objective function. The most common additional penalty terms in $\mathcal{J}^{\star}$ relate to:

- A measure of the rate of change of the control input, typically expressed as $\lambda_{1}\left\|\Delta u(t)^{2}\right\|$,

- The power losses related to the control action required of the PTO system, generally expressed as $\lambda_{2}\left\|u(t)^{2}\right\|$, and/or,

- Slack variables, used to convexify the optimisation problem [107], (Richter, 2011), which can be written as $\lambda_{3}\left\|\epsilon^{2}\right\|$ with $\epsilon=\left[\epsilon_{x} \epsilon_{\dot{x}}\right]$,

with $\lambda_{i} \in \mathbb{R}_{>0} \forall i$. It should be noted that, when using slack variables, in general, constraints on $\epsilon$ are added to the optimisation strategy (Richter, 2011). A summary, regarding the appearance of additional terms typically appearing in $\mathcal{J}^{\star}$, in the literature, is given in Table 2. Apart from the most common penalty terms shown in Table 2, several authors propose further modifications or alternative penalty terms. In Li and Belmont (2014a), a quadratic penalty term related to the buoyancy force is considered, with the purpose of guaranteeing the feasibility of the optimisation problem. Authors report that large incoming waves are more likely to cause such an infeasibility, thus the extra penalty term provides a further degree of freedom for tuning, to ensure that the constraint on the heave motion of the buoy can be satisfied for large incoming waves. In their study, the convexity of the objective function is analysed and guaranteed, as an improvement to a previous study (Li etal., 2012). Later on, an extension of the optimisation criteria developed in Li and Belmont (2014a), to an array of WECs, can be found in Li and Belmont (2014b), where the authors present a coordination-based distributed MPC (see e.g. Venkat, Hiskens, Rawlings, \& Wright, 2008). An additional study considering an array of WECs (with an optimisation criteria extended from Richter et al. (2013)) can be found in Oetinger et al. (2014), where a decentralized MPC strategy (see e.g. Bemporad \& Barcelli, 2010) is considered. Other studies, such as Tom and Yeung (2014) and de la Villa Jaén et al. (2014), consider a particular model for the PTO and, hence, a particular model for the absorbed power and the losses (if any are involved) in the objective function. In particular, Tom and Yeung (2014) studies the use of a permanent magnet generator as the PTO. An approximation of the power absorbed by the PTO is given by $P(t)=B_{g}(t) \dot{x}^{2}(t)$, where the magnitude of $B_{g}(t)$ (variable linear damping) can be manipulated either by altering the magnet coil gap width, or the applied electrical load (for more details, reader should refer to Tom \& Yeung, 2013). With this particular choice, the absorbed energy $\mathcal{J}_{2}$ becomes:

$\mathcal{J}=\int_{t}^{t+T_{h}} B_{g}(\tau) \dot{x}^{2}(\tau) d \tau$

Table 2

Considered additional terms in objective function.

\begin{tabular}{|c|c|}
\hline $\begin{array}{l}\text { Additional } \\
\text { term }\end{array}$ & References \\
\hline$\lambda_{1}\left\|\Delta u^{2}\right\|$ & $\begin{array}{l}\text { Cretel etal. (2010); Cretel et al. (2011); Li and Belmont (2014a); 2014b); Nguyen et al. (2016); Olaya et al. (2014); 2015); Tom and Yeung (2014); Tona et al. } \\
\text { (2015) }\end{array}$ \\
\hline$\lambda_{2}\left\|u^{2}\right\|$ & $\begin{array}{l}\text { Amann etal. (2015); Andersen et al. (2015); Cretel et al. (2011); de la Villa Jaén etal. (2014); Herber and Allison (2013); Kovaltchouk etal. (2015); Li and } \\
\text { Belmont (2014a); 2014b); Oetinger etal. (2014); O’Sullivan and Lightbody (2017); Richter et al. (2013, 2014) }\end{array}$ \\
\hline$\lambda_{3}\left\|\epsilon^{2}\right\|$ & Amann etal. (2015); Oetinger etal. (2014); Richter et al. $(2013,2014)$ \\
\hline
\end{tabular}



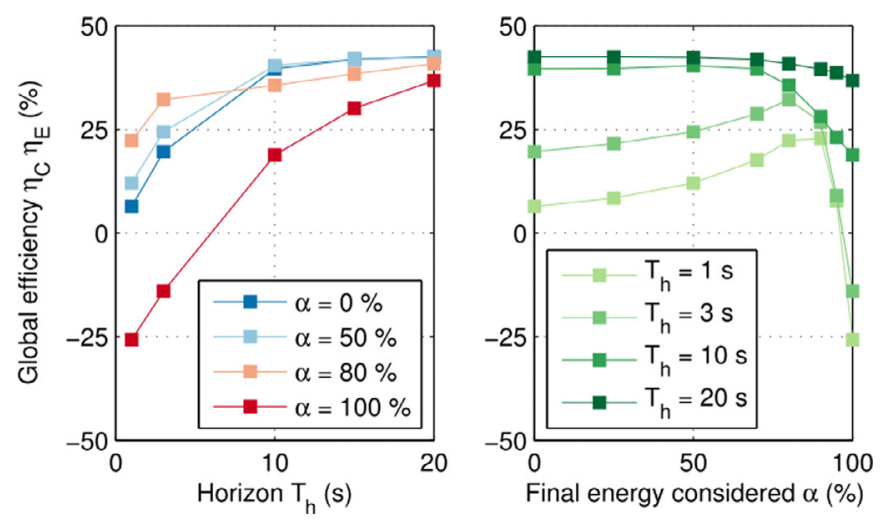

Fig. 10. Control performance for different time horizon $T_{h}$ and weight of final state mechanical energy $\alpha$ under a particular sea state (Kovaltchouk et al., 2015).

Tom and Yeung (2014) also considers a penalty term on the control input, which is expressed in terms of $B_{g}(t)$. In a subsequent study (Tom, 2014), same authors experimentally investigated the proposed MPC strategy using a scale model of the original WEC considered. In de la Villa Jaén et al. (2014) a MPC strategy applied to a point absorber, with a direct drive linear generator is presented. The model for the PTO system is as in Boldea and Nasar (1997). A model for the losses of the PTO system (copper losses, as described in Villa Jaén, García-Santana, \& Montoya-Andrade (2014)) is specifically considered in the optimisation process. Different objective function terms are considered in Kovaltchouk etal. (2015), where a weight for the endpoint state is considered. The authors report that without such a weight, the control strategy applied naturally tends to convert all the energy stored by the WEC at the end of the optimisationhorizon. The endpoint weight $\Phi$ is given as a portion of the mechanical energy that can be used after the horizon, namely $\Phi(x)=K \alpha E_{\text {mech }}(x)$. The influence of the coefficient $\alpha$ and the time horizon $T_{h}$ in their optimisationprocedure can be seen in Fig. 10. Note that the performance monotonically increases with $T_{h}$, since the authors assume perfect knowledge of the excitation force $\mathcal{F}_{\text {exc }}$. In Fig. 10, it can also be appreciated that there exists an optimal value of $\alpha$ between $0 \%$ and $100 \%$ that maximizes the performance of the control strategy. In Abraham and Kerrigan (2013), it is assumed that power is taken off through a damping force proportional to the velocity, written as $u^{*}(t)=-B_{u} u_{2}(t) \dot{x}(t)+u_{1}(t) G$, where $u_{1} \in[-1,1], G>0$ and $B_{u}$ is a constant damping coefficient, controlled proportionally through $u_{2}(t) \in[0,1]$. With this choice, the dynamics of the WEC remain bilinear (i.e. linear in the input and linear in the state, but not jointly linear in both) and the objective function remains,

$\mathcal{J}=\int_{t}^{t+T_{h}}-B_{u} u_{2}(\tau) \dot{x}^{2}(\tau)+u_{1}(\tau) G \dot{x}(\tau) d \tau$

A nonlinear efficiency term is considered in Tona etal. (2015), modelled as a function of the ideal instantaneous power $u(t) \dot{x}(t)$ :

$\eta(u(t) \dot{x}(t))=\left\{\begin{array}{lll}\eta_{0} & \text { if } & u(t) \dot{x}(t) \geq 0 \\ \frac{1}{\eta_{0}} & \text { if } & u(t) \dot{x}(t)<0\end{array} \quad 0 \leq \eta_{0} \leq 1\right.$

With the definition in (23), the absorbed energy is

$\mathcal{J}=-\int_{t}^{t+T_{h}} \eta(u(\tau) \dot{x}(\tau)) u(\tau) \dot{x}(\tau) d \tau$

Results of instantaneous power, under the optimal control formulation proposed in Tona et al. (2015), for different PTO efficiencies, can be seen in Fig. 11. An application of this same strategy (Tona et al., 2015), with extensive experimental results on a prototype device, can be found in Nguyen etal. (2016).

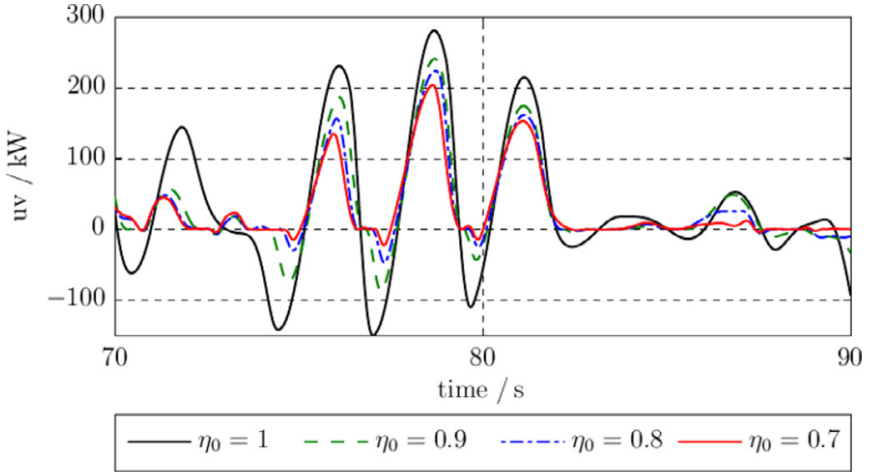

Fig. 11. Instantaneous power with optimal controls for different PTO efficiencies (Tona et al., 2015).

Although a considerable number of studies use the criterion defined in (20), there are some exceptions that present alternative objective functions. In Hals et al. (2011), the objective function attempts to maximize the difference between the incident power due to the wave excitation force and the total power losses expressed as

$\mathcal{J}=\int_{t}^{t+T_{h}}\left(P_{e}(\tau)-P_{r}(\tau)-P_{l}(\tau)\right) d \tau$

where $P_{e}(t)=f_{e}(t) \dot{x}(t)$ represents the excitation power, $P_{r}(t)=$ $f_{r}(t) \dot{x}(t)$ represents the radiated power and $P_{l}(t)$ the remaining power losses. Although the formulation in (25) takes into account these power losses, the optimisation process presented in Hals et al. (2011) neglects $P_{l}(t)$. An optimal velocity profile is obtained within this optimisation problem framework, which leads to a particular optimal control solution. Results regarding to the solution of such an optimisation problem, can be found in Fig. 12, where it can be seen that the optimal solution changes with varying wave height. Nevertheless, it is observed that, in all cases, the velocity peaks are in alignment with the excitation force peaks, agreeing with the frequency domain phase condition for unconstrained optimal motion (9b). The same objective function (25) is adopted later in Jama etal. (2014). Other approaches can be found in Brekken (2011); Jama etal. (2013) and Jama et al. (2014), where MPC is used for its original purpose of reference tracking. In Brekken (2011) and Jama etal. (2013), the optimal velocity profile is assumed known (as in (9)) and the objective function addresses the deviation of the device velocity from the given optimal velocity profile, similar to (15). A comprehensive analysis of constraint handling and possible infeasibilities of the solution with the traditional MPC approach can be found in Brekken (2011). In the case of Jama et al. (2013), continuous-time MPC is considered. Finally, in Jama et al. (2014), the authors of Jama et al. (2013) use MPC in order to achieve reference tracking, but the optimal profile is given by an optimisation procedure with objective function (25).

In summary, since the energy-maximising objective (20) is significantly different from the traditional "tracking" MPC formulation, a plethora of methods to obtain an efficient optimisation formulation can be found in the literature. Since the objective function $\mathcal{J}$, as defined in (19), is potentially non-convex, a variety of penalty terms $\mathcal{J}^{\star}$ can be found among different studies, mostly in an attempt to obtain a convex optimisation formulation, guaranteeing a unique global solution and real-time capability of the MPC optimal controller, since efficient algorithms can be used for the minimisation of a convex optimisation problem (see Section 5.7). Even performance functions differing significantly from (20) are proposed, pursuing the same energy-maximising objective from an alternative perspective. Regarding the penalty terms $\mathcal{J}^{\star}$, its important to notice that such a practice modifies the original 

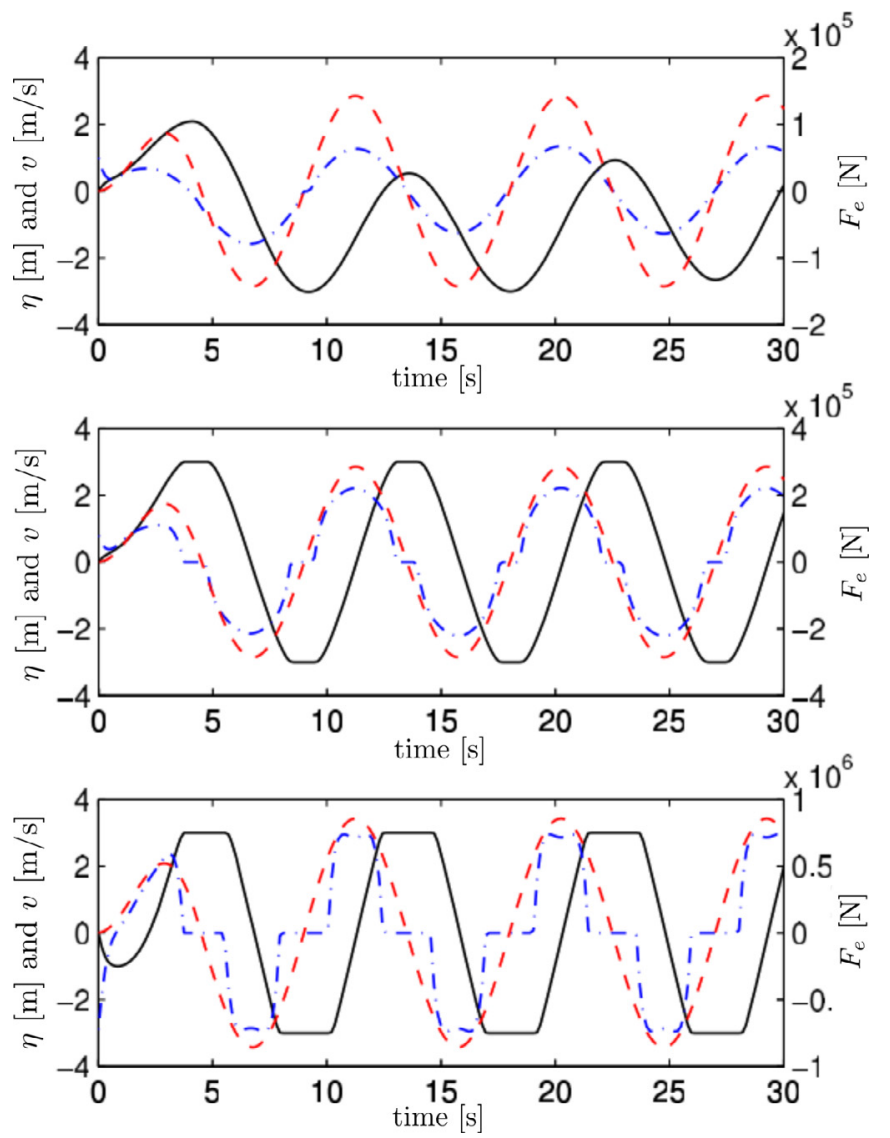

Fig. 12. Optimal velocity (blue, dash-dotted) and position (black, fully drawn) for the buoy excited by a regular wave of period 9s and varying wave height: $0.5 \mathrm{~m}$ (top), $1.0 \mathrm{~m}$ (middle) and $3.0 \mathrm{~m}$ (bottom). The red curve with scale on the righthand axis corresponds to the excitation force, which is a sine function. Heave amplitude is constrained to \pm 3 (Hals etal., 2011). (For interpretation of the references to colour in this figure legend, the reader is referred to the web version of this article.)

energy-maximising optimal control objective and, therefore, renders the formulation to be sub-optimal.

\subsection{Forecasting of $\mathcal{F}_{\text {exc }}$}

From (20), and the optimisation procedure, we noted that future knowledge of the excitation force $\mathcal{F}_{\text {exc }}(t)$ is required. This problem of short-term forecasting is analysed in Fusco and Ringwood (2012). The same authors present several wave prediction algorithms for real-time application in Fusco and Ringwood (2010). Additionally, further prediction algorithms are developed in Schoen, Hals, and Moan (2011). With reference to the MPC strategy, the excitation force $\mathcal{F}_{\text {exc }}$ prediction constitutes a fundamental issue that influences the selection of $T_{h}$ directly, since the uncertainty of the prediction gets larger over longer prediction horizons. For longer horizons, the control signal from the MPC algorithm is based on increasingly incorrect data, and the performance decreases compared with what is achieved with the exact excitation force. Hence, the loss in the accuracy of predictive algorithms limits the length of the prediction window for practical real-time usage (Fusco \& Ringwood, 2010). However, there is a natural synergy between the prediction requirement for a device, and the predictability of the sea in which it is designed to operate (Fusco \& Ringwood, 2012).

Although most studies assume that the future wave excitation force is known over the time horizon considered (ostensibly to focus on the control problem, in the spirit of the

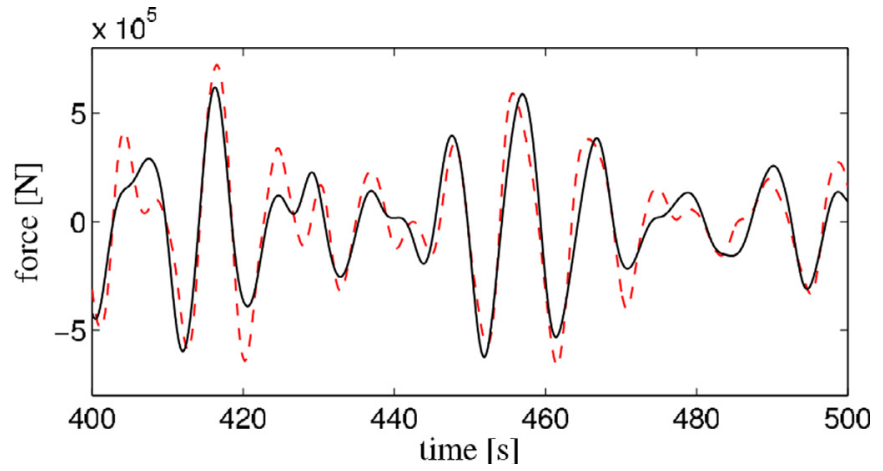

Fig. 13. The force signal is predicted $2.2 \mathrm{~s}$ ahead (dashed, red curve) and compared to true values (fully drawn, black curve) (Hals et al., 2011). (For interpretation of the references to colour in this figure legend, the reader is referred to the web version of this article.)

separation principle Bertsekas, 2000), several authors employing MPC strategies cited in this review also developed and implemented combined estimation and forecasting methodologies. Such is the case of, for example, Hals etal. (2011), where the excitation force is predicted using an augmented Kalman filter based on a time-varying damped harmonic oscillator model of the wave process. An example of the predictor's performance (Hals etal., 2011) can be found in Fig. 13. The same study presents performance results on a MPC controller, with exact and predicted excitation force. Brekken (2011), uses a linear Kalman filter to obtain an estimate of the excitation force and an auto-regressive (AR) model, with an adaptive least squares strategy (Fusco \& Ringwood, $2010)$, is used for predict future values of $\mathcal{F}_{\text {exc }}(t)$. A combination of a Kalman filter and an AR model is also presented in Andersen et al. (2015). In Li and Belmont (2014a); Li et al. (2012) and Li and Belmont (2014b) a different approach is used, based on deterministic sea wave prediction (DSWP) (Morris, Zienkiewicz, \& Belmont, 1998). In both de la Villa Jaén etal. (2014) and Tona etal. (2015), an AR model is used to predict $\mathcal{F}_{\text {exc }}(t)$. Finally, in Cavaglieri, Bewley, and Previsic (2015), an alternative approach is considered, where an ensemble Kalman filter (which assimilates data from a Doppler wave radar), combined with a pseudospectral wave model, is used to forecast the excitation force (see also the author's thesis (Cavaglieri, 2016) for a detailed description of the method).

In summary, since future knowledge of the excitation force $\mathcal{F}_{\text {exc }}$ is required in order to compute the optimal control policy within the optimisation horizon, different forecasting methodologies have been reported. We note that the requirement for future values of $\mathcal{F}_{\text {exc }}$ for the computation of the optimal control sequence, creates a downward pressure on the allowable length of the optimisation window. Most of the studies assume perfect knowledge of the excitation force over the time horizon considered (somewhat akin to the separation principle used in traditional optimal control/estimation problems), leading to possibly non-realistic results. Therefore, some degree of uncertainty on $\mathcal{F}_{\text {exc }}$ should be considered in order to evaluate the performance of any WEC optimal controller with a higher accuracy. The impact of forecast errors in $\mathcal{F}_{\text {exc }}$ on WEC control has been, to some extent, considered in Fusco and Ringwood (2011).

\subsection{Optimisation}

Most of the studies reviewed represent the optimisation procedure as a Quadratic Programming (QP) problem. A quadratic programming problem is a special case of Nonlinear Programming (NLP) problems, in which the objective functional is a quadratic form and the constraints are linear (Boyd \& Vandenberghe, 2004). Examples of this approach are Andersen etal. (2015); 
Brekken (2011); Cretel et al. (2010); Cretel et al. (2011); Hals et al. (2011); Jama etal. (2013); 2014); Li and Belmont (2014a); 2014b); Oetinger etal. (2014); Olaya etal. (2014); 2015); Richter etal. (2014) and de la Villa Jaén etal. (2014). In such a case, the problem can be solved iteratively by active set strategies or interior point methods, where each iteration requires the solution of an equalityconstrained QP problem (Boyd \& Vandenberghe, 2004). A few exceptions should be noted, where the optimisation problem is no longer regarded as a QP problem. In Amann etal. (2015); Richter etal. (2013); Tom and Yeung (2014) and Tona etal. (2015), the problem is regarded as NLP. Authors in Richter et al. (2013) claim that a nonlinear interior-point strategy (Wächter \& Biegler, 2006) can be used to solve such an optimisation problem. They also report that online computation is not possible with the solver used in their study, although they declare that the objective of their approach is not focused on real-time applicability, but rather to the qualitative performance of NMPC using a nonlinear WEC model. A similar approach is also used in Amann etal. (2015); Tom and Yeung (2014) and Tona et al. (2015).

A distinction needs to be made at this point, since some studies do not consider the process of solving the optimisation problem (20) as a nonlinear program. The numerical optimisation procedure used in the MPC strategy to solve the optimal control problem (in this case, (20)), can be categorised as a direct method. In a direct method, the state and/or the control input are discretised and the optimal control problem is transcribed to a NLP. MPC-like algorithms, detailed in Section 6, are also classified as direct methods. However, some receding horizon algorithms developed in the literature, fall into the category of indirect methods.

Indirect methods are based on the calculus of variations (Liberzon, 2011) and Pontryagin's maximum (or minimum) principle, Pontryagin (1987). The optimal solution is, in general, derived in two steps: the first step formulates the optimal problem as a Two-Point Boundary Value Problem (TPBVP) (Liberzon, 2011), while the second step solves the TPBVP, which can be carried out analytically or numerically (Liberzon, 2011). One of the most common approaches to solve the TPBVP numerically is the shooting method (Rao, 2009). The main difference between direct and indirect methods is that direct methods attempt to directly minimise (or maximise) the objective function $\mathcal{J}$, whereas indirect methods attempt to solve the necessary conditions of optimality (which must be derived analytically for each problem). Studies reviewed that consider indirect methods (within a receding horizon strategy) are Abraham and Kerrigan (2013); Li et al. (2012) and Kovaltchouk et al. (2015). In Li etal. (2012), the authors do not assume (or guarantee) the convexity of the objective function. Pontryagin's minimum principle is used to conclude that a nearly optimal control for the problem is of a bang-bang nature (see Liberzon, 2011 for more details). The problem is solved using Dynamic Programming (DP), which is a multi-stage decision process, based on Bellman's principle of optimality (Bellman, 1956; Larson \& Casti, 1978). A forward dynamic programming algorithm is implemented, within the receding horizon principle. In Abraham and Kerrigan (2013), a computationally improved and globally convergent variation of the projected gradient method (see e.g. Bertsekas, 1999) is developed and compared with an interior point solver to demonstrate its performance. Finally, in Kovaltchouk etal. (2015), a mixed state control constraint is presented which complicates the application of Pontryagin's principle (De Pinho, Vinter, \& Zheng, 2001). In order to avoid the mixed state control constraint, the authors use a barrier function, retaining only the constraint on the control input. After applying Pontryagin's principle, a multishooting method is used to compute the optimal control policy (Diehl, Bock, Diedam, \& Wieber, 2006).

In summary, the optimisation procedure involved in the optimal control formulation (20) can be dealt with in a variety of ways.
Despite the fact that most of the studies regard the problem as a QP, several direct and indirect methods are also applied to the wave energy control problem. The main disadvantage of indirect methods is that the necessary conditions for optimality must be derived analytically, (which increases in difficulty when, for example, considering nonlinearities in the WEC model), while direct methods transcribe the optimisation problem as a NLP, and attempt to minimise (20) directly. Indeed, indirect methods for nonlinear systems can be applied to classical problems and some special weakly nonlinear low dimensional systems, as further described in Von Stryk and Bulirsch (1992). Nevertheless, direct methods, in general, produce less accurate solutions than indirect methods. The choice of an optimisation procedure is then strictly related to the formulation of Eq. (20), and is strongly dependent on the model of the WEC considered.

\section{MPC-like algorithms: spectral and pseudospectral techniques}

Control algorithms based on spectral methods offer an interesting alternative to MPC, as they can be used to solve optimal control problems under constraints using a specific parameterisation of the solution (Garg, Hager, \& Rao, 2011). Spectral methods have shown have appealing computational aspects, offering the possibility of scaling in complexity/performance by changing the number of approximating basis functions. Both spectral and pseudospectral are included in the family of discretisation methods based on the Mean Weighted Residuals (MWR), where both state and control variables are parameterised. The main idea behind MWR is to assume that the state and the control input can be approximated by a linear combination of specific basis functions $\xi_{k}^{u}(t)$ and $\xi_{k}^{x}(t)$ (notation adopted from Bacelli \& Ringwood, 2015), as

$$
\begin{aligned}
& x_{i}(t) \approx x_{i}^{N_{x}} \doteq \sum_{k=1}^{N_{x}} \hat{x}_{i k} \xi_{k}^{x}(t) \\
& u_{i}(t) \approx u_{i}^{N_{u}} \doteq \sum_{k=1}^{N_{u}} \hat{u}_{i k} \xi_{k}^{u}(t)
\end{aligned}
$$

where $x_{i}, x_{i}^{N_{x}}, u_{i}, u_{i}^{N_{u}}$ are the $i$ th components of the vectors $x, x^{N_{x}}$, $u$ and $u^{N_{u}}$ respectively. Assuming that the dynamics of the system are given by $\dot{x}=f(x, u, t)$, the resulting residual function is defined as,

$R(X, U, t)=\dot{x}^{N_{x}}-f(X, U, t)$

where the vectors $X$ and $U$ contain the coefficients $\hat{x}_{i k}$ and $\hat{u}_{i k}$ respectively. For any value of $U$, the corresponding vector $X$ is obtained by solving the following system of equations

$\left\langle R_{i}(X, U, t) ; \phi_{j}(t)\right\rangle=0 \quad$ for $i, j=1, \ldots, N_{x}$

where $\langle f, g\rangle=\int_{\Gamma} f(t) g(t) d t$ is the inner product with a unity weight function and the test functions $\phi_{j}(t)$ are orthogonal under the same inner product. If the test functions $\phi_{j}(t)$ are elements of the same set as the basis functions approximating the state, that is $\phi(t)_{j}=\xi_{k}^{x}(t)$, then the method is known as a spectral or Galerkin method. If, on the other hand, the test functions are translated Dirac-delta functions $\delta\left(t-t_{j}\right)$, then the method takes the name of pseudospectral or collocation method. Unlike MPC which, in most of the applications, effectively uses $\mathrm{ZOH}$ functions to approximate the optimal solution, spectral and pseudospectral methods are (generally) based on functions defined over the complete control horizon, i.e. have global, rather than local, support. Different choices for the basis functions are studied in Genest and Ringwood (2017) and can be appreciated in Fig. 14. Another important aspect relating to spectral methods is that they provide a simplification of the convolution integral associated with the radiation force (3), when the velocity is approximated within the selected basis. 
a

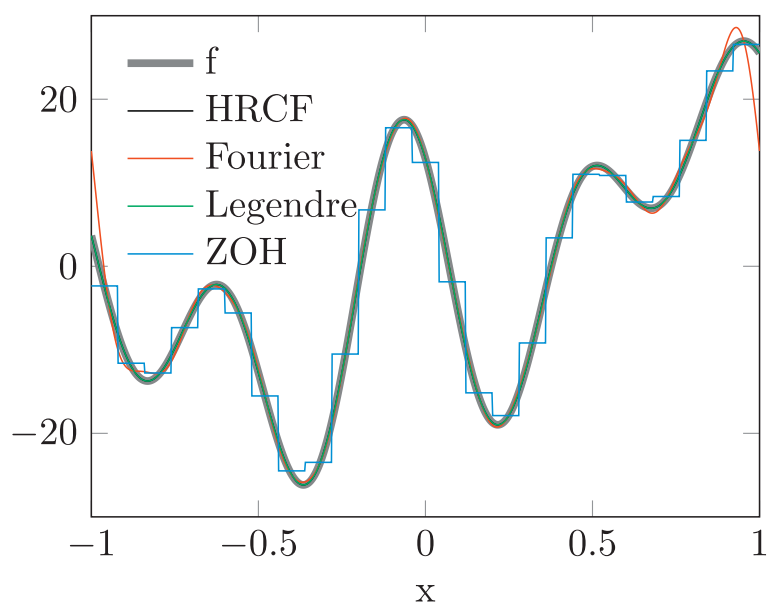

b

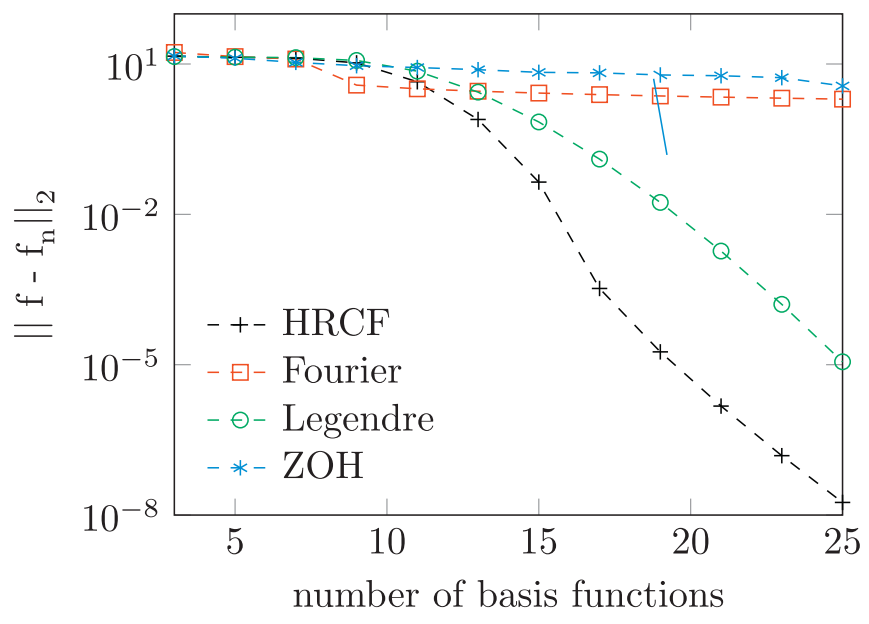

Fig. 14. Approximation of a nonperiodic function using different sets of functions. ZOH: Zero-Order Hold; HRCF: Half-Range Chebyshev Fourier functions; Legendre: Legendre polynomials; Fourier: Trigonometric Fourier functions (Genest \& Ringwood, 2017) - (b) Approximation errors for same sets of orthogonal function sets.

Moreover, Bacelli and Ringwood (2015) show that the computation involving the numerical integration of the convolution integral can be carried out offline, thus significantly reducing the computational load when solving the NLP.

\subsection{Spectral methods}

The first application of spectral methods in wave energy can be found in Bacelli etal. (2011), where the device considered is a self-reacting point absorber. The objective function adopted for the optimisation procedure is the same as (19). The orthonormal basis chosen $\left(\xi_{k}^{x, u}(t)\right)$ is in terms of Fourier functions, inspired by the oscillatory nature of the problem. To solve the constrained optimisation problem, the algorithm uses the penalty method (Wright \& Nocedal, 1999). Due to the properties of Fourier functions, Bacelli et al. (2011) also present a suboptimal solution using a 2-norm that is more efficient in computational terms. Further development and analysis of constraints within the spectral framework can be found in Bacelli and Ringwood (2011). In Westphalen, Bacelli, Balitsky, and Ringwood (2011), the same authors use a similar approach to develop a control strategy for an array of single-body devices of cylindrical shape in heave motion only. Two control algorithms are tested: one operating on an individual device level, called the independent device controller (IC), and a global array controller (GC). IC does not take the interactions from the other devices into account and optimises the motion based on the total incident and diffracted waves only, while GC explicitly takes the hydrodynamic coupling of all devices into account and seeks a global optimum. The metric used to evaluate the performance of the strategy is the q-factor (Thomas \& Evans, 1981). Such results are extended by the same authors in Bacelli and Ringwood (2013), where several array configurations and different body geometries are studied. The procedure is defined for a general WEC array ( $n$ devices), with an objective function given by

${ }^{n} \mathcal{J}=-\sum_{k=1}^{n} \int_{t}^{t+T_{h}} u(\tau) \dot{x}_{k}(\tau) d \tau$

Perfect knowledge of future wave elevation is assumed, in order to isolate the effects of control on the total energy produced. Also, an adaptive constraint approach is used in the case of IC, in order to avoid violations caused by the control model, which neglects part of the hydrodynamic interaction between WECs. Both IC and GC are regarded as a $\mathrm{QP}$, and are solved using an active set algorithm. The authors present three possible layouts and three different geometries. A sensitivity analysis of the relative IC/GC performance with respect to position variation of an array element is studied in Bacelli, Balitsky, and Ringwood (2013). Also, the case with an incorrect estimation of the excitation force is considered under the same control strategy (Bacelli et al., 2013). Another noteworthy study, concerning arrays of WECs based on spectral methods, is Garcia-Rosa, Bacelli, and Ringwood (2015b), where the integration of optimal array design and control design is studied. Finally, in Garcia-Rosa, Bacelli, and Ringwood (2015a), the impact of device motion and input constraints in the optimised design of a WEC is studied, using spectral techniques and following the procedures from Bacelli and Ringwood (2015).

\subsection{Pseudospectral methods}

An early appearance of pseudospectral techniques in the context of control of WECs can be found in Herber and Allison (2013). A co-design approach, with PTO restrictions, is considered. The direct transcription method used is the Radau pseudospectral method with Legrende-Gauss-Radau collocation points and a $h p$-adaptive mesh refinement algorithm (Fahroo \& Ross, 2008), where specific General Pseudospectral Optimisation Software (Rao etal., 2010) is used. The objective function selected in the optimisation process is the same as in (19). Later on, Bacelli and Ringwood (2015) present a general mathematical framework for the solution of the WEC control problem, using both spectral and pseudospectral methods, where the Galerkin method (in conjunction with trigonometric polynomials), applied to a heaving point absorber, is extensively discussed. Simulations of the strategy, with both regular and irregular waves, are given. In Bacelli and Ringwood (2014), a nonlinear application of the pseudospectral method is studied for a flap-type device. The nonlinearity is due to the moment of the drag force (Journée \& Massie, 2000). The objective function, for the case of a rotating flap, involves the angular momentum $\theta(t)$ and the torque applied by the PTO:

${ }^{\theta} \mathcal{J}=\int_{t}^{t+T_{h}} u(\tau) \dot{\theta}(\tau) d \tau$

The basis for the pseudospectral expansion is a zero-mean trigonometric polynomial (truncated Fourier series). The optimisation problem is solved using Sequential Quadratic Programming (SQP) (Nocedal \& Wright, 2006). The study developed in Bacelli, Genest, and Ringwood (2015) expands these result further, considering constraints and a non-ideal PTO model. A generic efficiency 
curve is used in this study (which is a function of the load factor only), adding nonlinear terms to the objective function (30). In Paparella and Ringwood (2017a), pseudospectral methods, based on Bacelli and Ringwood (2015), are applied to a hinge-barge wave energy converter (Fig. 7), for which the dynamical model is originally derived and validated by the same authors as in Paparella et al. (2016). In their study (Paparella \& Ringwood, 2017a), the linear dynamical model of the device is derived with two different formulations: a Differential and Algebraic Equation (DAE) formulation and an Ordinary Differential Equations (ODE) representation. The basis chosen for the expansion is a truncated Fourier series. The optimisation process in Paparella etal. (2016) considers the energy-maximising objective function as defined in (19). Due to the non-convex formulation obtained, Paparella etal. (2016) describes a method to convexify the objective function, by introducing a weight on the control power inside the objective function. Constraints are considered in relation to both the motion of the device, and the control inputs. Results are presented using both the DAE and ODE representations for regular and irregular waves. Moreover, a pseudospectral passive controller (i.e. incorporating a unidirectional power flow constraint) is developed and compared to the original active (bidirectional power flow) formulation.

A recent study, presenting an alternative to pseudospectral methods, termed a Shape-Based (SB) approach, can be found in Abdelkhalik etal. (2016). The main difference between SB and pseudospectral methods is that SB only approximates one of the states of the system (velocity $\dot{x}$ ) rather than all the states and the control input, as in the pseudospectral approach, reducing the computational cost involved in the optimisation process. SB assumes a Fourier basis expansion only for the velocity of the buoy, with unknown coefficients. The frequencies in the velocity expansion are extracted from a (assumed available) prediction of the excitation force. In this study, two models are considered: a simplified version, using the same dynamical model as Li etal. (2012); and a "performance model" with a more accurate radiation force description (detailed in Coe \& Bull (2014)). The objective function considered is the same as in (19) and the optimisation algorithm, to obtain the optimal shape of the state, is the interior point algorithm. In their results, several comparisons with different strategies (developed in Cretel etal. (2011); Li etal. (2012) and Bacelli and Ringwood (2015)) are given, in order to test the proposed strategy.

\subsection{Summary of spectral and pseudospectral methods}

In summary, spectral and pseudospectral methods have shown to be appealing for the wave energy control formulation since both propose a specific parameterisation of the solution, which is well matched to the oscillatory nature of the problem. As can be appreciated in Fig. 14, (and already mentioned in Section 5.4), spectral and pseudospectral methods capture the dynamics of the system in an efficient and precise way, using a basis function expansion. This efficiency facilitates the use of the original objective function (19) in the optimisation process, while maintaining real-time capabilities. Nevertheless, most formulations are based on trigonometric polynomials and, hence, compute periodic solutions, which represents a drawback in a receding-horizon context.

\subsection{Receding horizon pseudospectral control}

Motivated by the fact that the aforementioned spectral and pseudospectral algorithms all calculate periodic solutions, wave energy researchers began to apply Receding Horizon Pseudospectral Control (RHPSC) in order to overcome this limitation. Moreover, recent studies have shown the utility of developing a RHPSC strategy to solve optimal control problems in real-time such as Williams (2004) and Fahroo and Ross (2008). A first approach, within the

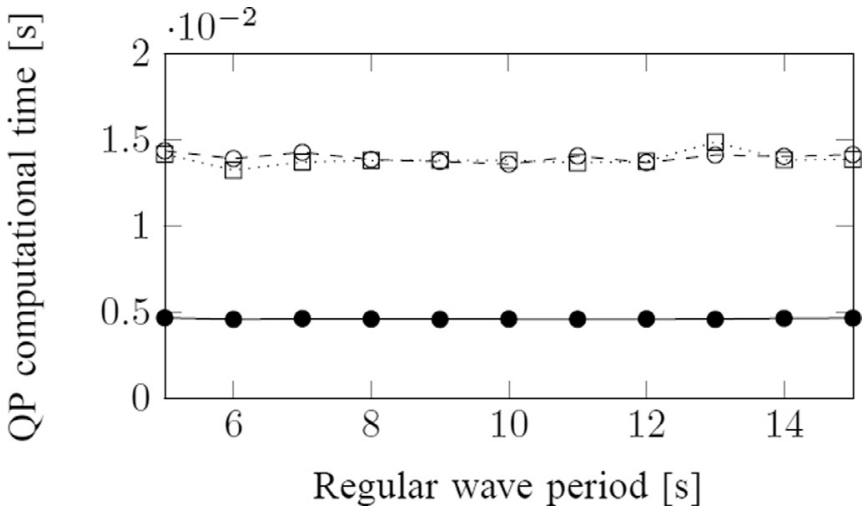

Fig. 15. QP computational time for $\operatorname{MPC}(\circ, \square)$ and RHPSC algorithm $(\cdot)$ for different regular wave periods. Different approximations for the radiation forces are used for this comparison namely NTNU Toolbox ( $\square$ ) and Prony's method (o) (Genest \& Ringwood, 2016a).

WEC context, can be found in Li (2015), where the differential flatness of the WEC model is combined with pseudospectral methods to develop a RHPSC strategy. In this study, the convolution is not approximated in pseudospectral form, as mentioned in Section 6. Instead, the classical approach, by means of a state-space approximation (16), as documented in Section 5.3, is used. The chosen basis functions for the RHPSC framework are Lagrangian polynomials. Using the differential flatness property of the model (see Sira-Ramirez and Agrawal, 2004 for a differential flatness formal definition), the objective function is approximated with a nonconvex function with a differentially flat output as the only optimisation variable. Simulations are performed to test the computational burden of the algorithm developed in Li (2015), considering the differential flatness of the system, combined with pseudospectral methods. The corresponding NLP is solved using SQP methods. Another study can be found in Genest and Ringwood (2017), where a real-time RHPSC formulation is developed based on HalfRange Chebyshev Fourier (HCRF) polynomials (Huybrechs, 2010) as basis functions, which can represent harmonic signals in the application domain, and also deal efficiently with the signal truncation effects associated with a receding horizon formulation. Moreover, such a basis fulfils the requirements to simulate both the transient and steady-state responses of the device. The WEC used in Genest and Ringwood (2017) is a flap-type device. The NLP is solved using SQP. Finally, a comparison between an MPC strategy and a RHPSC formulation, for a heaving point absorber, is given in two studies, namely (Genest \& Ringwood, 2016a) and Genest and Ringwood (2016b), where the chosen MPC algorithm is the same as in Cretel et al. (2011) and the RHPSC strategy is as in Genest and Ringwood (2017). In Genest and Ringwood (2016a), the difference between computational time of both strategies, under constrained optimisation, is compared. Simulations are given with regular and irregular waves. Their results (see Fig. 15) suggest that the computational burden is at least 3 times smaller for the RHPSC algorithm than for an MPC strategy. For the comparison, Genest and Ringwood (2016a) uses different algorithms for the radiation force computation in MPC strategies, namely the NTNU Toolbox (Perez \& Fossen, 2009) and Prony's method (Duclos, Clément, Chatry et al., 2001). Time series of the position, velocity and control input obtained from the WEC, controlled with both MPC and RHPSC algorithms, without path constraints, can be seen in Fig. 16. All proposed trajectories follow the optimal path determined in Falnes (2002).

In Paparella and Ringwood (2017b), a RHSPC strategy with HRCF functions is applied to a multibody hinge-barge WEC (as shown in Fig. 7), originally derived in Paparella and Ringwood (2017a). In this study, the authors develop an equivalent reduced dynamical 


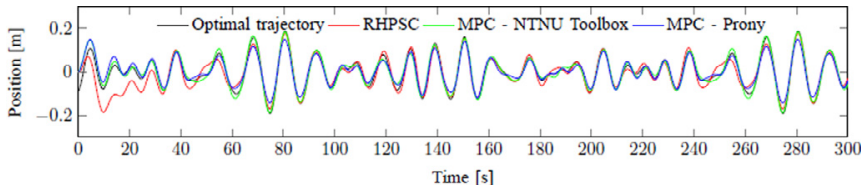

Time $[s]$
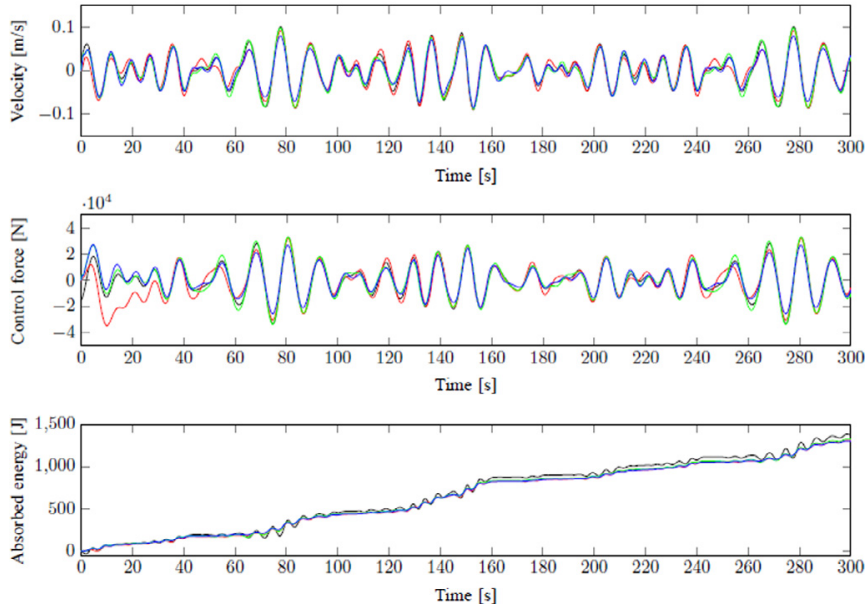

Fig. 16. Normalised absorbed power and QP computational time for MPC and RHPSC algorithms for a given irregular sea state (Genest \& Ringwood, 2016a).

model for the control formulation, which is described in terms of the relative pitch rotations, reducing the number of variables involved and, therefore, reducing the computational effort required to compute the optimal control policy. An alternative convex formulation of the optimisation problem, where the objective function is expressed in terms of the difference between the excitation force power and the dissipated power from radiated waves and viscous forces, is presented. This approach is similar to that considered in Hals etal. (2011) and, therefore, specific to the objective function in (25). An active and a passive controller are developed using RHSPC methods in Paparella and Ringwood (2017b). Performance of both controllers are presented in the results, for both monochromatic and polychromatic waves.

In summary, several studies consider pseudospectral methods within a receding-horizon framework, by adopting non-periodic problem-specific basis functions, such as HRCF functions. The use of HRCF functions shows a major improvement regarding the computational burden associated with the optimisation process, as can be seen in Fig. 15, where the computational time is at least 3 times smaller for the RHSPC formulation compared to MPC.

A perspective might be considered where both MPC, which uses time domain discretisation of a state-space model, and pseudospectral (or spectral) control, which expands the system variables onto an orthonormal basis, involve the discretisation of a continuous-time problem into a discrete space, in which the optimisation problem can be computationally solved. Despite the fact that, at first glance, these disparate discretisation philosophies appear to be quite different, there are ways in which both approaches can be viewed within an integrated framework. Key to such a perspective is Fig.14(a), where the $\mathrm{ZOH}$ approximation can be considered as the use of a set of rectangular basis functions, within a pseudospectral framework. In fact, such rectangular functions, or the trapezoidal 'basis' functions associated with a first-order hold, constitute an orthonormal basis (Jiang, Schoufelberger, Thoma, \& Wyner, 1992). However, a dichotomy is observed that the "basis" functions associated with state-space discretisation provide only local support, while the Fourier or HRCF functions associated with the pseudospectral WEC controllers provide global support. This is somewhat analogous to the case of nonlinear interpolation using artificial neural networks, where radial basis networks and support vector machines provide local approximation capability while, for example, multi-layer perceptrons utilise interpolating functions with global support (Haykin, Haykin, Haykin, \& Haykin, 2009). In terms of choosing between the two broad families of discretisation, it could be argued that the Fourier/HRCF function basis provides a basis more suited to the WEC control application, given their improved computational properties (for at least a similar level of energy conversion) over rectangular/trapezoidal functions, as evidenced by Fig.15. However, the global support of Fourier functions leads to difficulties with a receding-horizon formulation, necessitating the alternative HRCF functions, which could also be deemed to have relatively local support.

\section{Discussion and further directions}

MPC strategies, as optimal formulations, have been successfully implemented in wave energy, for maximisation of converted energy. They have the enormous advantage of handling constraints in a natural way, within the optimisation process, which is fundamental in order to ensure that the control strategy respects the physical limitations of the device. However, the computational burden of MPC algorithms can render them unsuitable for real-time applications, potentially in the case of WEC arrays. Spectral and pseudospectral methods are slowly entering the field, in an attempt to diminish the computational time, with RHPSC. Nevertheless, much work has to be done to establish a real-time strategy based on a repeated optimisation process. In order to provide a critical comparison between studies, concerning both MPC and MPC-like algorithms, Table 4 provides the reader with a comprehensive summary of the main characteristics highlighted and discussed in this review study. A list of abbreviations, used in Table 4, can be found in Table 3 .

On the other hand, most of the results are based on linear hydrodynamic models, as can be seen in Fig. 17; NMPC must be further developed in order to apply such strategies successfully to real devices. A recent study, highlighting the importance of using nonlinear models, when computing the optimal control policy in the wave energy context, can be found in Mérigaud and Ringwood (2017). Another aspect that merits further development relates to optimisation of the entire energy conversion process, since it depends on multiple power conversion stages (Penalba Retes, Giorgi, \& Ringwood, 2015). Regarding the objective function, which is at the heart of the optimisation process, several studies have added terms in order to obtain a convex quadratic problem and reduce the computational challenge to compute an optimal solution. One must notice that such a practice affects the original energy maximising optimal criterion, and such a trade-off has to be further analysed in practical situations. However, spectral techniques can handle the original objective function with a relatively low computational burden, making them a competitive alternative to MPC. This can be further appreciated in Fig. 18, where the percentage of MPC studies concerning the original energy-maximising objective function (19) is shown, and is considerably lower than in the case of spectral or pseudospectral methods.

Another aspect that requires further research in wave energy applications concerns stability. Despite lot of development regarding MPC stability for the classical reference tracking problem (Rawlings \& Muske, 1993; Rossiter \& Kouvaritakis, 1993; Zheng \& Morari, 1994), such results are not straightforwardly applicable to the wave energy case, since the objective function is significantly different. Stability analysis is a fundamental property regarding the design of any real controller; therefore, such analysis should be taken into account in future research studies. It is important to notice that even when the optimisation algorithm finds a solution, 
Table 3

Comparison between the main characteristics of studies reviewed.

\begin{tabular}{|c|c|c|c|c|c|c|c|c|c|c|c|c|c|c|c|c|c|c|}
\hline \multirow[t]{3}{*}{ Reference } & \multicolumn{3}{|c|}{ Dynamical Model } & \multicolumn{4}{|c|}{ Constraints } & \multirow[t]{3}{*}{$\mathcal{F}_{\text {exc }}$ conv. } & \multirow[t]{3}{*}{ Discretisation } & \multicolumn{6}{|c|}{ Obj. Function } & \multicolumn{3}{|c|}{ Opt. Problem } \\
\hline & $\overline{\mathrm{LTI}}$ & LTV & $\mathrm{NL}$ & $\overline{A V}$ & $\mathrm{~V} \mathrm{U}$ & $\mathrm{U} \quad \mathrm{DU}$ & P Ref & & & $\overline{\mathrm{E}}$ & DU & $\mathrm{U}$ & Sv & Ref & $\bar{T}$ & $\overline{\mathrm{QP}}$ & NLP & $\overline{\text { IN }}$ \\
\hline & $\overline{\mathrm{SA}}$ & & & & & & & & & & & & & & & & & \\
\hline Li et al. (2012) & - & & & - & • & • & & Not considered & $\mathrm{ZOH}$ & $\cdot$ & & & & & & & & $\cdot$ \\
\hline Richter et al. (2014) & $\cdot$ & & & . $\quad$ & - . & $\cdot$ & & Damping & $\mathrm{ZOH}$ & $\cdot$ & & - & - & & & - & & \\
\hline Richter et al. (2013) & & & $\cdot$ & - $\cdot$ & - $\cdot$ & • & & Damping & Taylor $2 \frac{\text { nd }}{2}$ order & $\cdot$ & & $\cdot$ & • & & & & - & \\
\hline Li and Belmont (2014a) & $\cdot$ & & & • & • & $\cdot$ & & Damping & $\mathrm{ZOH}$ & $\cdot$ & - & - & & See Section 5.5 & & - & & \\
\hline Li and Belmont (2014b) & - & & & - & • & $\cdot \cdot$ & & Damping & $\mathrm{ZOH}$ & $\cdot$ & - & - & & See Section 5.5 & & - & & \\
\hline Olaya et al. (2014) & - & & & - $\cdot$ & - $\cdot$ & • & & Implicit & $\mathrm{ZOH}$ & $\cdot$ & • & & & & & - & & \\
\hline Olaya et al. (2015) & - & & & & • & $\cdot$ & & Implicit & $\mathrm{ZOH}$ & $\cdot$ & - & & & & & - & & \\
\hline Tom and Yeung (2014) & & - & & - & • & $\cdot$ & & SS $-3 \frac{r d}{-}$ order & Trapezoidal Rule & $\cdot$ & - & & & $(21)$ & & & - & \\
\hline Amann etal. (2015) & & & • & $\cdot \cdot$ & - $\cdot$ & • & & Not considered & Taylor $2 \frac{\text { nd }}{\underline{4}}$ order & $\cdot$ & & $\cdot$ & $\cdot$ & & & & $\cdot$ & \\
\hline Kovaltchouk et al. (2015) & - & & & & • & $\cdot$ & - & SS $-4^{\text {th }}$ order & Trapezoidal Rule & $\cdot$ & & $\cdot$ & & See Section 5.5 & & & & $\cdot$ \\
\hline Oetinger et al. (2014) & $\cdot$ & & & $\cdot \quad \cdot$ & - $\cdot$ & $\cdot$ & & Not considered & $\mathrm{ZOH}$ & $\cdot$ & & $\cdot$ & $\cdot$ & & & $\cdot$ & & \\
\hline Andersen et al. (2015) & - & & & & • & $\cdot$ & & Not specified & $\mathrm{ZOH}$ & $\cdot$ & & $\cdot$ & & & & $\cdot$ & & \\
\hline Tona et al. (2015) & $\cdot$ & & & & • & $\cdot$ & & SS $-5^{\text {th }}$ order & Tustin rule & $\cdot$ & $\cdot$ & & & $(24)$ & & & $\cdot$ & \\
\hline Nguyen et al. (2016) & \multicolumn{18}{|c|}{ Experimental application of Tona etal. (2015) } \\
\hline Brekken (2011) & $\cdot$ & & & $\cdot \cdot$ & $\cdot$ & $\cdot$ & & Damping & Not specified & & & & & & $\cdot$ & $\cdot$ & & \\
\hline Jama et al. (2014) & $\cdot$ & & & $\cdot$ & & $\cdot$ & & SS $-4^{\text {th }}$ order & ZOH Laguerre F. & & & & & $(25)$ & $\cdot$ & $\cdot$ & & \\
\hline O’Sullivan and Lightbody (2017) & • & & & $\cdot \cdot$ & - $\cdot$ & • & See Section 2.2 & SS $-4^{\text {th }}$ order & $\mathrm{FOH}$ & $\cdot$ & & $\cdot$ & & & & • & $\cdot$ & \\
\hline Hals et al. (2011) & - & & & - & • & $\cdot$ & & SS $-4^{\text {th }}$ order & $\mathrm{ZOH}$ & & & & & $(25)$ & & - & & \\
\hline Cretel et al. (2010) & - & & & • & & & & SS - 6 $\frac{\text { th }}{\text { order }}$ & $\mathrm{ZOH}$ & $\cdot$ & • & & & & & - & & \\
\hline Cretel et al. (2011) & • & & & $\cdot \cdot$ & • & & & SS $-5^{\text {th }}$ order & $\mathrm{FOH}$ & $\cdot$ & $\cdot$ & $\cdot$ & & & & $\cdot$ & & \\
\hline de la Villa Jaén et al. (2014) & $\cdot$ & & & $\cdot$ & • & $\cdot$ & & SS $-5^{\text {th }}$ order & $\mathrm{FOH}$ & $\cdot$ & & $\cdot$ & & & & $\cdot$ & & \\
\hline Cavaglieri et al. (2015) & $\cdot$ & & & $\cdot \quad \cdot$ & $\cdot$. & $\cdot$ & & Not specified & Not specified & $\cdot$ & & & & & & $\cdot$ & & \\
\hline Soltani et al. (2014) & $\cdot$ & & & & • & $\cdot$ & & Not specified & $\mathrm{ZOH}$ & - & & & & & & $\cdot$ & & \\
\hline Jama et al. (2013) & - & & & & • & $\cdot$ & & SS $-4^{\text {th }}$ order & Laguerre F. & & & & & & $\cdot$ & $\cdot$ & & \\
\hline Abraham and Kerrigan (2013) & $\cdot$ & & & & $\cdot$ & $\cdot$ & & SS $-3 \frac{r d}{d}$ order & Implicit & & & & & $(22)$ & & & & $\cdot$ \\
\hline \multicolumn{19}{|c|}{ MPC-Like Algorithms } \\
\hline Li (2015) & & & $\cdot$ & $\cdot$ & • & • & & SS $-3 \frac{r d}{r}$ order & PS (Lagrangian) & $\cdot$ & & & & & & & $\cdot$ & \\
\hline Bacelli et al. (2011) & $\cdot$ & & & $\cdot$ & & & & $\mathrm{SP}$ (Fourier) & SP (Fourier) & $\cdot$ & & & & & & & $\cdot$ & \\
\hline Genest and Ringwood (2017) & $\cdot$ & & & $\cdot \quad \cdot$ & - $\cdot$ & $\cdot$ & & PS (HRCF) & PS (HRCF) & $\cdot$ & & & & & & & $\cdot$ & \\
\hline Herber and Allison (2013) & - & & & $\cdot$ & • & $\cdot$ & See Section 2.2 & Damping & PS (LGR) & - & & $\cdot$ & & & & & $\cdot$ & \\
\hline Westphalen et al. (2011) & - & & & $\cdot$ & & & & SP (Fourier) & SP (Fourier) & $\cdot$ & & & & & & & $\cdot$ & \\
\hline Bacelli and Ringwood (2013) & $\cdot$ & & & $\cdot$ & • & $\cdot$ & & SP (Fourier) & SP (Fourier) & $\cdot$ & & & & (29) & & $\cdot$ & & \\
\hline Bacelli et al. (2013) & $\cdot$ & & & $\cdot$ & $\cdot$ & $\cdot$ & & $\mathrm{SP}$ (Fourier) & SP (Fourier) & $\cdot$ & & & & & & $\cdot$ & & \\
\hline Bacelli et al. (2015) & & & $\cdot$ & $\cdot \quad \cdot$ & $\cdot \cdot$ & $\cdot$ & $\cdot$ & PS (Fourier) & PS (Fourier) & $\cdot$ & & & & & & & $\cdot$ & \\
\hline Abdelkhalik etal. (2016) & - & & & $\cdot$ & • & $\cdot$ & & SS $-8^{\text {th }}$ order & SB (Fourier) & - & & & & & & & $\cdot$ & \\
\hline Bacelli and Ringwood (2015) & - & & & $\cdot$ & • & $\cdot$ & & SP/PS (Fourier) & SP/PS (Fourier) & - & & & & & & - & $\cdot$ & \\
\hline Bacelli and Ringwood (2014) & & & • & & & & & PS (Fourier) & PS (Fourier) & • & & & & $(30)$ & & & - & \\
\hline Garcia-Rosa et al. (2015b) & - & & & & & & & SP (Fourier) & SP (Fourier) & $\cdot$ & & & & & & $\cdot$ & & \\
\hline Garcia-Rosa et al. (2015a) & $\cdot$ & & & $\cdot$ & • & $\cdot$ & & SP (Fourier) & SP (Fourier) & $\cdot$ & & & & & & $\cdot$ & & \\
\hline Genest and Ringwood (2016a) & • & & & $\cdot \cdot$ & - $\cdot$ & • & & PS (HRCF) & PS (HRCF) & $\cdot$ & & & & & & & $\cdot$ & \\
\hline Genest and Ringwood (2016b) & - & & & $\cdot \cdot$ & - $\cdot$ & • & & PS (HRCF) & PS (HRCF) & $\cdot$ & & & & & & & $\cdot$ & \\
\hline Paparella and Ringwood (2017a) & - & & & $\cdot \cdot$ & - $\cdot$ & $\cdot$ & & PS (Fourier) & PS (Fourier) & - & & & & & & . & & \\
\hline Paparella and Ringwood (2017b) & - & & & - & - $\cdot$ & $\cdot$ & & PS (HRCF) & PS (HRCF) & - & & & & & & - & & \\
\hline
\end{tabular}

this does not guarantee stability of the overall system, that is, optimality does not imply stability.

Robustness of MPC controllers for wave energy applications needs to be considered, since little work has been done in this area. Robust analysis of applied strategies must be considered, since additional modelling errors will arise due to unmodelled dy- namics, nonlinearities, etc., especially considering the challenge of developing computationally tractable hydrodynamic models of adequate fidelity (Giorgi etal., 2016). While robust control is a mature field in tracking problems, it has received a little attention (apart from, for example Fusco \& Ringwood, 2014) in the wave energy application. Naturally, a further possible direction may be 
Table 4

List of abbreviations used in Table 3.

\begin{tabular}{|c|c|c|}
\hline Section in table & Term & \\
\hline \multirow{5}{*}{ Dynamical Model } & LTI & Linear time-invariant \\
\hline & LTV & Linear time-varying \\
\hline & NL & Nonlinear \\
\hline & S & Single device \\
\hline & A & Array configuration \\
\hline \multirow{5}{*}{ Constraints } & A & Amplitude \\
\hline & $\mathrm{V}$ & Velocity \\
\hline & $\mathrm{U}$ & Control input \\
\hline & DU & Rate of change of the control input \\
\hline & $\mathrm{P}$ & Maximum power \\
\hline \multirow{3}{*}{$\mathcal{F}_{\text {exc }}$ conv. } & SS & State-Space \\
\hline & SP & Spectral \\
\hline & PS & Pseudospectral \\
\hline \multirow[t]{2}{*}{ Discretisation } & SB & Shape-Based \\
\hline & $\mathrm{E}$ & Original energy-maximising objective function \\
\hline \multirow{4}{*}{ Objective function } & DU & $\begin{array}{l}\text { Penalty term related to rate of change of the } \\
\text { control input }\end{array}$ \\
\hline & $\mathrm{U}$ & Penalty term related to the control input \\
\hline & Sv & Slack variables \\
\hline & $\mathrm{T}$ & Trajectory tracking objective function \\
\hline \multirow{3}{*}{ Optimisation problem } & QP & Quadratic programming \\
\hline & NLP & Nonlinear programming \\
\hline & IN & Indirect method \\
\hline
\end{tabular}

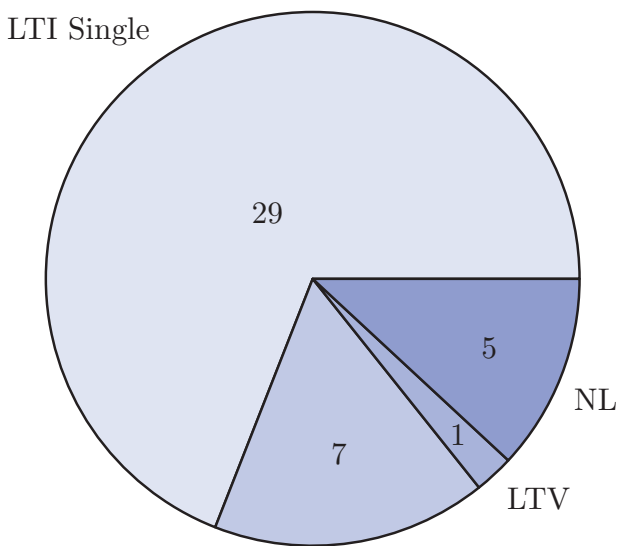

LTI Array

Fig. 17. Type of dynamical model considered in literature reviewed. References: LTI $\equiv$ linear time-invariant; LTV $\equiv$ linear time-varying; $\mathrm{NL} \equiv$ nonlinear. Data extracted from Table 4.

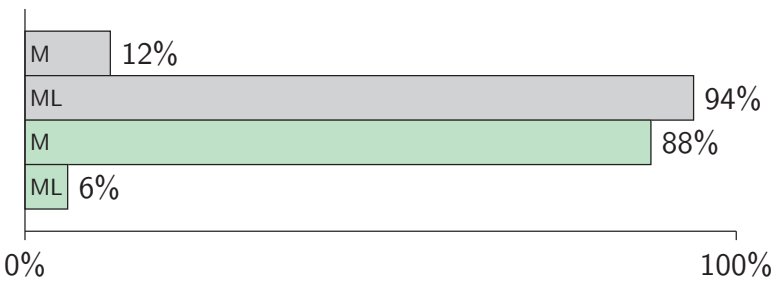

Fig. 18. Percentage of MPC (M) and MPC-Like (ML) algorithms in the literature reviewed that: consider the original energy-maximising objective function (19) (represented by grey coloured bars); consider a modified or different objective function (represented by green coloured bars). Data extracted from Table 4. (For interpretation of the references to colour in this figure legend, the reader is referred to the web version of this article.)

provided by examining the applicability of robust MPC (Camacho \& Alba, 2013) controllers to wave energy conversion.

To date, the majority of WEC controllers have been evaluated in simulation, with varying degrees of simulation fidelity, with a few notable exceptions, such as the Pelamis WEC (Yemm, Pizer, \& Retzler, 2002), the Wavestar WEC (Kramer, Marquis, \& Frigaard, 2011) and the SEAREV WEC (Cordonnier, Gorintin, De Cagny, Clé- ment, \& Babarit, 2015). One important issue is that, frequently, WEC controllers are simulated with a model identical to that upon which the controller is designed, completely masking (with the exception of some minor numerical inaccuracies) any sensitivity of the control system to modelling errors. However, ideally, WEC controllers should be evaluated in more realistic wet tests, using a wave tank or, at the very least, a numerical wave tank (NWT) (Davidson, Cathelain, Guillemet, Le Huec, \& Ringwood, 2015) with a high-fidelity hydrodynamic solver based on, for example, computational fluid dynamics (CFD) or smooth particle hydrodynamics (SPH). One or two studies are now beginning to emerge using such high-fidelity simulations (Davidson, Genest, Ringwood, 2017) and some proposals are currently underway to compare WEC controllers under a standardized tank-testing protocol (Ringwood et al., 2017).

\section{Acknowledgment}

This material is based upon works supported by Science Foundation Ireland under grant no. 13/IA/1886.

\section{References}

Abdelkhalik, O., Robinett, R., Zou, S., Bacelli, G., Coe, R., Bull, D., et al. (2016). On the control design of wave energy converters with wave prediction. Journal of Ocean Engineering and Marine Energy, 2(4), 473-483.

Abraham, E., \& Kerrigan, E. C. (2013). Optimal active control and optimization of a wave energy converter. IEEE Transactions on Sustainable Energy, 4(2), 324-332.

Amann, K. U., Magaña, M. E., \& Sawodny, O. (2015). Model predictive control of a nonlinear 2-body point absorber wave energy converter with estimated state feedback. IEEE Transactions on Sustainable Energy, 6(2), 336-345.

Andersen, P., Pedersen, T. S., Nielsen, K. M., \& Vidal, E. (2015). Model predictive control of a wave energy converter. In Conference on control applications, Sydney (pp. 1540-1545). IEEE.

Babarit, A., \& Clément, A. H. (2006). Optimal latching control of a wave energy device in regular and irregular waves. Applied Ocean Research, 28(2), 77-91.

Babarit, A., Duclos, G., \& Clément, A. H. (2004). Comparison of latching control strategies for a heaving wave energy device in random sea. Applied Ocean Research, 26(5), 227-238.

Babarit, A., Guglielmi, M., \& Clément, A. H. (2009). Declutching control of a wave energy converter. Ocean Engineering, 36(12), 1015-1024.

Babarit, A., Mouslim, H., Guglielmi, M., \& Clément, A. H. (2008). Simulation of the Searev wave energy converter with a by-pass control of its hydraulic power take off. In World renewable energy congress, Glasgow (pp. 1004-1009).

Bacelli, G. (2014). Optimal control of wave energy converters. Maynooth University Ph.D. thesis..

Bacelli, G., Balitsky, P., \& Ringwood, J. V. (2013). Coordinated control of arrays of wave energy devices-benefits over independent control. IEEE Transactions on Sustainable Energy, 4(4), 1091-1099.

Bacelli, G., Genest, R., \& Ringwood, J. V. (2015). Nonlinear control of flap-type wave energy converter with a non-ideal power take-off system. Annual Reviews in Control, 40, 116-126.

Bacelli, G., \& Ringwood, J. V. (2011). A geometrical interpretation of force and position constraints in the optimal control of wave energy devices. In 9th European wave and tidal energy conference, Southampton.

Bacelli, G., \& Ringwood, J. V. (2013). Constrained control of arrays of wave energy devices.. International Journal of Marine Energy, 3, 53-69.

Bacelli, G., \& Ringwood, J. V. (2014). Nonlinear optimal wave energy converter control with application to a flap-type device. IFAC Proceedings Volumes, 47(3), 7696-7701.

Bacelli, G., \& Ringwood, J. V. (2015). Numerical optimal control of wave energy converters. IEEE Transactions on Sustainable Energy, 6(2), 294-302.

Bacelli, G., Ringwood, J. V., \& Gilloteaux, J.-C. (2011). A control system for a self-reacting point absorber wave energy converter subject to constraints. In 18th IFAC world congress, Milano: vol. 44 (pp. 11387-11392). Elsevier.

Bellman, R. (1956). Dynamic programming and Lagrange multipliers. Proceedings of the National Academy of Sciences, 42(10), 767-769.

Bemporad, A., \& Barcelli, D. (2010). Decentralized model predictive control. In Networked control systems (pp. 149-178). Springer.

Bertsekas, D. P. (1999). Nonlinear programming. Athena Scientific Belmont.

Bertsekas, D. P. (2000). Dynamic programming and optimal control (2nd). Athena Scientific.

Boldea, I., \& Nasar, S. A. (1997). Linear electric actuators and generators. In IEEE international electric machines and drives conference record, Wisconsin (pp. MA1-1) IEEE.

Boyd, S., \& Vandenberghe, L. (2004). Convex optimization. Cambridge University Press.

Brekken, T. K. (2011). On model predictive control for a point absorber wave energy converter. In Powertech, trondheim (pp. 1-8). IEEE. 
Budal, K., \& Falnes, J. (1975). A resonant point absorber of ocean-wave power. Nature, 256(5517), 478-479.

Budal, K., \& Falnes, J. (1977). Optimum operation of improved wave-power converter. Marine Science Communications, 3(2), 133-150.

Budal, K., \& Falnes, J. (1980). Interacting point absorbers with controlled motion. Power from sea waves (pp. 381-399).

Camacho, E. F., \& Alba, C. B. (2013). Model predictive control. Springer Science \& Business Media.

Cavaglieri, D. (2016). New numerical methods for computational fluid dynamics, forecast and control. Ph.D. thesis.

Cavaglieri, D., Bewley, T. R., \& Previsic, M. (2015). Model predictive control leveraging ensemble Kalman forecasting for optimal power take-off in wave energy conversion systems. In American control conference, Chicago (pp. 5224-5230). IEEE.

Clarke, D., Mohtadi, C., \& Tuffs, P. (1987). Generalized predictive control-part I. The basic algorithm. Automatica, 23(2), 137-148.

Clément, A., \& Maisondieu, C. (1993). Comparison of time domain control law for a piston wave absorber. In European wave energy symposium, Scotland (pp. 117-122).

Coe, R. G., \& Bull, D. L. (2014). Nonlinear time-domain performance model for a wave energy converter in three dimensions. In Oceans 2014, St. John's (pp. 1-10). IEEE.

Cordonnier, J., Gorintin, F., De Cagny, A., Clément, A., \& Babarit, A. (2015). SEAREV: Case study of the development of a wave energy converter. Renewable Energy, $80,40-52$.

Cretel, J., Lewis, A., Lightbody, G., \& Thomas, G. (2010). An application of model predictive control to a wave energy point absorber. IFAC Proceedings Volumes, 43(1), 267-272.

Cretel, J. A., Lightbody, G., Thomas, G. P., \& Lewis, A. W. (2011). Maximisation of energy capture by a wave-energy point absorber using model predictive control. IFAC Proceedings Volumes, 44(1), 3714-3721.

Cummins, W. (1962). The impulse response function and ship motions. Technical Report. DTIC Document.

Cutler, C. R., \& Ramaker, B. L. (1980). Dynamic matrix control: A computer control algorithm. In Joint automatic control conference, San Francisco 17 (p. 72).

Davidson, J., Cathelain, M., Guillemet, L., Le Huec, T., \& Ringwood, J. (2015). Implementation of an openfoam numerical wave tank for wave energy experiments. In 11th European wave and tidal energy conference, Nantes.

Davidson, J., Genest, R., \& Ringwood, J. (2017). Adaptive control of a wave energy converter simulated in a numerical wave tank. In 12th European wave and tidal energy conference, Cork.

de la Villa Jaén, A., Santana, A. G., et al. (2014). Considering linear generator copper losses on model predictive control for a point absorber wave energy converter. Energy Conversion and Management, 78, 173-183.

de O. Falcão, A. F. (2010). Wave energy utilization: A review of the technologies. Renewable and Sustainable Energy Reviews, 14(3), 899-918.

De Pinho, M., Vinter, R., \& Zheng, H. (2001). A maximum principle for optimal control problems with mixed constraints. IMA Journal of Mathematical Control and Information, 18(2), 189-205.

Diehl, M., Bock, H. G., Diedam, H., \& Wieber, P.-B. (2006). Fast direct multiple shooting algorithms for optimal robot control. In Fast motions in biomechanics and robotics (pp. 65-93). Springer.

Drew, B., Plummer, A. R., \& Sahinkaya, M. N. (2009). A review of wave energy converter technology. Proceedings of the Institution of Mechanical Engineers, Part A: Journal of Power and Energy, 223(8), 887-902.

Duclos, G., Clément, A. H., \& Chatry, G. (2001). Absorption of outgoing waves in a numerical wave tank using a self-adaptive boundary condition. International Journal of Offshore and Polar Engineering, 11(3), 168-175.

Edenhofer, O., Pichs-Madruga, R., Sokona, Y., Seyboth, K., Kadner, S., Zwickel, T., et al. (2011). Renewable energy sources and climate change mitigation: Special report of the intergovernmental panel on climate change. Cambridge University Press.

Eidsmoen, H. (1996). Optimum control of a floating wave-energy converter with restricted amplitude. Journal of Offshore Mechanics and Arctic Engineering. Transactions of the ASME, 118(2), 96-101.

Eidsmoen, H. (1996). Simulation of a slack-moored heaving-buoy wave-energy converter with phase control. Technical report. Norwegian University of Science and Technology, Norway.

Eidsmoen, H. (1996). Simulation of a tight-moored amplitude limited heaving-buoy wave-energy converter with phase control. Technical report. Norwegian University of Science and Technology, Norway.

Eidsmoen, H. (1998). Tight-moored amplitude-limited heaving-buoy wave-energy converter with phase control. Applied Ocean Research, 20(3), 157-161.

Evans, D. (1979). Some theoretical aspects of three-dimensional wave-energy absorbers. In Proceedings of the first symposium on wave energy utilization, Gothenburg (pp. 77-106).

Evans, D. (1981). Maximum wave-power absorption under motion constraints. Applied Ocean Research, 3(4), 200-203.

Evans, D. (1981). Power from water waves. Annual Review of Fluid Mechanics, 13(1), $157-187$.

Fahroo, F., \& Ross, I. M. (2008). Pseudospectral methods for infinite-horizon nonlinear optimal control problems. Journal of Guidance, Control, and Dynamics, 31(4), 927-936.

Falnes, J. (1980). Radiation impedance matrix and optimum power absorption for interacting oscillators in surface waves. Applied Ocean Research, 2(2), 75-80.
Falnes, J. (2002). Ocean waves and oscillating systems: Linear interactions including wave-energy extraction. Cambridge University Press.

Feng, Z., \& Kerrigan, E. C. (2013). Latching control of wave energy converters using derivative-free optimization. In 52nd annual conference on decision and control, Firenze (pp. 7474-7479). IEEE.

Feng, Z., \& Kerrigan, E. C. (2014). Declutching control of wave energy converters using derivative-free optimization. IFAC Proceedings Volumes, 47(3), 7647-7652.

Feng, Z., \& Kerrigan, E. C. (2015). Latching-declutching control of wave energy converters using derivative-free optimization. IEEE Transactions on Sustainable Energy, 6(3), 7474-7479.

Franklin, G. F., Powell, J. D., \& Workman, M. L. (1998). Digital control of dynamic systems: vol.3. Menlo Park: Addison-Wesley.

French, M. (1979). A generalized view of resonant energy transfer. Journal of Mechanical Engineering Science, 21(4), 299-300.

Fusco, F., \& Ringwood, J. (2011). A model for the sensitivity of non-causal control of wave energy converters to wave excitation force prediction errors. In 9th European wave and tidal energy conference, Southampton.

Fusco, F., \& Ringwood, J. V. (2010). Short-term wave forecasting for real-time control of wave energy converters. IEEE Transactions on Sustainable Energy, 1(2), 99-106.

Fusco, F., \& Ringwood, J. V. (2012). A study of the prediction requirements in realtime control of wave energy converters. IEEE Transactions on Sustainable Energy, $3(1), 176-184$.

Fusco, F. \& Ringwood, J. V. (2014). Hierarchical robust control of oscillating wave energy converters with uncertain dynamics. IEEE Transactions on Sustainable Energy, 5(3), 958-966.

Garcia, C. E., Prett, D. M., \& Morari, M. (1989). Model predictive control: Theory and practice-A survey. Automatica, 25(3), 335-348.

Garcia-Rosa, P. B., Bacelli, G., \& Ringwood, J. V. (2015). Control-informed geometric optimization of wave energy converters: The impact of device motion and force constraints. Energies, 8(12), 13672-13687.

Garcia-Rosa, P. B., Bacelli, G., \& Ringwood, J. V. (2015). Control-informed optimal array layout for wave farms. IEEE Transactions on Sustainable Energy, 6(2), 575-582.

Garg, D., Hager, W. W., \& Rao, A. V. (2011). Pseudospectral methods for solving infinite-horizon optimal control problems. Automatica, 47(4), 829-837.

Genest, R., Bonnefoy, F., Clément, A. H., \& Babarit, A. (2014). Effect of non-ideal power take-off on the energy absorption of a reactively controlled one degree of freedom wave energy converter. Applied Ocean Research, 48, 236243.

Genest, R., \& Ringwood, J. V. (2016). A critical comparison of model-predictive and pseudospectral control for wave energy devices. Journal of Ocean Engineering and Marine Energy, 2(4), 485-499.

Genest, R., \& Ringwood, J. V. (2016). Receding horizon pseudospectral optimal control for wave energy conversion. In UKACC 11th international conference on control, Belfast (pp. 1-6). IEEE.

Genest, R., \& Ringwood, J. V. (2017). Receding horizon pseudospectral control for energy maximization with application to wave energy devices. IEEE Transactions on Control Systems Technology, 25(1), 29-38.

Gieske, P. (2007). Model predictive control of a wave energy converter: Archimedes wave swing. Delft University of Technology (TU Delft) Master's thesis.

Giorgi, G., Retes, M. P., \& Ringwood, J. V. (2016). Nonlinear hydrodynamic models for heaving buoy wave energy converters. In Asian wave and tidal energy conference, Singapore (pp. 144-153).

Guenther, D. A., Jones, D., \& Brown, D. G. (1979). An investigative study of a wave-energy device. Energy, 4(2), 299-306.

Hals, J., Bjarte-Larsson, T., \& Falnes, J. (2002). Optimum reactive control and control by latching of a wave-absorbing semisubmerged heaving sphere. In 21st international conference on offshore mechanics and arctic engineering, Oslo (pp. 415-423). American Society of Mechanical Engineers.

Hals, J., Falnes, J., \& Moan, T. (2011). Constrained optimal control of a heaving buoy wave-energy converter. Journal of Offshore Mechanics and Arctic Engineering, 133(1), 011401.

Hansen, R. H., \& Kramer, M. M. (2011). Modelling and control of the wavestar prototype. In 9th European wave and tidal energy conference, Southampton (p. 10).

Haykin, S. S., Haykin, S. S., Haykin, S. S., \& Haykin, S. S. (2009). Neural networks and learning machines: vol. 3. Pearson.

Herber, D. R., \& Allison, J. T. (2013). Wave energy extraction maximization in irregular ocean waves using pseudospectral methods. In International design engineering technical conferences and computers and information in engineering conference, Portland. American Society of Mechanical Engineers. V03AT03A018

Hoskin, R., \& Nichols, N. (1987). Optimal strategies for phase control of wave energy devices. Utilization of ocean waves-Wave to energy conversion (pp. 184-199).

Huybrechs, D. (2010). On the Fourier extension of nonperiodic functions. SIAM Journal on Numerical Analysis, 47(6), 4326-4355.

Jama, M., Wahyudie, A., Assi, A., \& Noura, H. (2013). Controlling heaving wave energy converter using function-based model predictive control technique. In 25th Chinese control and decision conference, Guiyang (pp. 2705-2710). IEEE.

Jama, M., Wahyudie, A., Assi, A., \& Noura, H. (2014). Function-based model predictive control approach for maximum power capture of heaving wave energy converters. In Icrega14-renewable energy: Generation and applications (pp. 299-313). Springer.

Jiang, Z., Schoufelberger, W., Thoma, M., \& Wyner, A. (1992). Block pulse functions and their applications in control systems. Springer-Verlag New York, Inc.

Journée, J., \& Massie, W. (2000). Offshore hydromechanics. Delft University of Technology. 
Khalil, H. (1996). Nonlinear systems. Prentice Hall.

Koca, K., Kortenhaus, A., Oumeraci, H., Zanuttigh, B., Angelelli, E., Cantu, M., et al. (2013). Recent advances in the development of wave energy converters. In 10th European wave and tidal energy conference, Aalborg (pp. 2-5).

Korde, U. A. (2000). Control system applications in wave energy conversion. In Oceans 2000 MTS/IEEE conference and exhibition, Providence: vol.3 (pp. 1817-1824). IEEE.

Kovaltchouk, T., Rongere, F., Primot, M., Aubry, J., Ahmed, H. B., \& Multon, B. (2015). Model predictive control of a direct wave energy converter constrained by the electrical chain using an energetic approach. In 11th European wave and tidal energy conference, Nantes.

Kramer, M., Marquis, L., \& Frigaard, P. (2011). Performance evaluation of the wavestar prototype. In 9th European wave and tidal energy conference, Southampton.

Kristiansen, E., Hjulstad, Å., \& Egeland, O. (2005). State-space representation of radiation forces in time-domain vessel models. Ocean Engineering, 32(17), 2195-2216.

Larson, R. E., \& Casti, J. L. (1978). Principles of dynamic programming. Dekker.

Li, G. (2015). Nonlinear model predictive control of a wave energy converter based on differential flatness parameterisation. International Journal of Control, 90(1), $1-10$.

Li, G., \& Belmont, M. R. (2014). Model predictive control of sea wave energy converters-Part I: A convex approach for the case of a single device. Renewable Energy, 69, 453-463.

Li, G., \& Belmont, M. R. (2014). Model predictive control of sea wave energy converters-Part II: The case of an array of devices. Renewable Energy, 68, 540-549.

Li, G., Weiss, G., Mueller, M., Townley, S., \& Belmont, M. R. (2012). Wave energy converter control by wave prediction and dynamic programming. Renewable Energy, 48, 392-403.

Li, S., Lim, K. Y., \& Fisher, D. G. (1989). A state space formulation for model predictive control. AIChE Journal, 35(2), 241-249.

Liberzon, D. (2011). Calculus of variations and optimal control theory: A concise introduction. Princeton University Press.

Maciejowski, J. M. (2002). Predictive control: With constraints. Pearson Education.

Mérigaud, A., \& Ringwood, J. V. (2017). Optimal trajectories, nonlinear models and constraints in wave energy device control. In Submitted to proceedings of the 20th IFAC world congress, Toulouse.

Mork, G., Barstow, S., Kabuth, A., \& Pontes, M. T. (2010). Assessing the global wave energy potential. In ASME 2010 29th international conference on ocean, offshore and arctic engineering, Vancouver (pp. 447-454). American Society of Mechanical Engineers.

Morris, E., Zienkiewicz, H., \& Belmont, M. (1998). Short term forecasting of the sea surface shape. International Shipbuilding Progress, 45(444), 383-400.

Mundon, T., Murray, A. F., Hallam, J., \& Patel, L. N. (2005). Causal neural control of a latching ocean wave point absorber. In International conference on artificial neural networks, Warsaw (pp. 423-429). Springer.

Naito, S., \& Nakamura, S. (1986). Wave energy absorption in irregular waves by feedforward control system. In Hydrodynamics of ocean wave-energy utilization (pp. 269-280). Springer.

Nguyen, H.-N., Sabiron, G., Tona, P., Kramer, M. M., \& Sanchez, E. V. (2016). Experimental validation of a nonlinear MPC strategy for a wave energy converter prototype. In 35th international conference on ocean, offshore and arctic engineering, Busan. American Society of Mechanical Engineers V006T09A019.

Nie, R., Scruggs, J., Chertok, A., Clabby, D., Previsic, M., \& Karthikeyan, A. (2016). Optimal causal control of wave energy converters in stochastic waves-accommodating nonlinear dynamic and loss models. International Journal of Marine Energy, 15, 41-55.

Nielsen, S. R., Zhou, Q., Kramer, M. M., Basu, B., \& Zhang, Z. (2013). Optimal control of nonlinear wave energy point converters. Ocean Engineering, 72, 176-187.

Nocedal, J., \& Wright, S. J. (2006). Sequential quadratic programming. Springer.

Oetinger, D., Magaña, M. E., \& Sawodny, O. (2014). Decentralized model predictive control for wave energy converter arrays. IEEE Transactions on Sustainable Energy, 5(4), 1099-1107.

Olaya, S., Bourgeot, J.-M., \& Benbouzid, M. (2014). Optimal control for a self-reacting point absorber: A one-body equivalent model approach. In International power electronics and application conference and exposition, Shanghai (pp. 332-337). IEEE.

Olaya, S., Bourgeot, J.-M., \& Benbouzid, M. (2015). On the generator constraint design of a wave energy converter at a pre-sizing stage. In 11th European wave and tidal energy conference, Nantes Paper10B3-3-1.

O'Sullivan, A. C., \& Lightbody, G. (2017). Co-design of a wave energy converter using constrained predictive control. Renewable Energy, 102, 142-156.

Paparella, F., Bacelli, G., Paulmeno, A., Mouring, S. E., \& Ringwood, J. V. (2016). Multibody modelling of wave energy converters using pseudo-spectral methods with application to a three-body hinge-barge device. IEEE Transactions on Sustainable Energy, 7(3), 966-974.

Paparella, F., \& Ringwood, J. V. (2017). Optimal control of a three-body hinge-barge wave energy device using pseudospectral methods. IEEE Transactions on Sustainable Energy, 8(1), 200-207.

Paparella, F., \& Ringwood, J. V. (2017). Receding horizon pseudo-spectral control for energy maximization of a $1 / 25$ th scale hinge-barge wave energy converter. In Submitted to proceedings of the 12th European wave and tidal energy conference, Cork.

Pelc, R., \& Fujita, R. M. (2002). Renewable energy from the ocean. Marine Policy, 26(6), 471-479.
Penalba Retes, M., Giorgi, G., \& Ringwood, J. V. (2015). A review of non-linear approaches for wave energy converter modelling. In 11th European wave and tidal energy conference, Nantes.

Pérez, T., \& Fossen, T. I. (2008). Time- vs. frequency-domain identification of parametric radiation force models for marine structures at zero speed. Modeling, Identification and Control, 29(1), 1-19.

Perez, T., \& Fossen, T. I. (2009). A Matlab toolbox for parametric identification of radiation-force models of ships and offshore structures. Modeling, Identification and Control, 30(1), 1.

Polinder, H., Damen, M. E., \& Gardner, F. (2004). Linear PM generator system for wave energy conversion in the AWS. IEEE Transactions on Energy Conversion, 19(3), 583-589.

Pontryagin, L. S. (1987). Mathematical theory of optimal processes. CRC Press.

Rao, A. V. (2009). A survey of numerical methods for optimal control. Advances in the Astronautical Sciences, 135(1), 497-528.

Rao, A. V., Benson, D. A., Darby, C., Patterson, M. A., Francolin, C., Sanders, I., \& Huntington, G. T. (2010). Algorithm 902: GPOPS, a Matlab software for solving multiple-phase optimal control problems using the Gauss pseudospectral method. ACM Transactions on Mathematical Software (TOMS), 37(2), 22.

Rawlings, J. B., \& Muske, K. R. (1993). The stability of constrained receding horizon control. IEEE Transactions on Automatic Control, 38(10), 1512-1516.

Richalet, J., Rault, A., Testud, J., \& Papon, J. (1976). Algorithmic control of industrial processes. In 4th IFAC symposium on identification and system parameter estimation, Tbilisi (pp. 1119-1167). IFAC.

Richalet, J., Rault, A., Testud, J., \& Papon, J. (1978). Model predictive heuristic control: Applications to industrial processes. Automatica, 14(5), 413-428.

Richter, M. (2011). Different model predictive control approaches for controlling point absorber wave energy converters. Master's thesis.

Richter, M., Magaña, M. E., Sawodny, O., \& Brekken, T. K. (2013). Nonlinear model predictive control of a point absorber wave energy converter. IEEE Transactions on Sustainable Energy, 4(1), 118-126.

Richter, M., Magaña, M. E., Sawodny, O., \& Brekken, T. K. (2014). Power optimisation of a point absorber wave energy converter by means of linear model predictive control. IET Renewable Power Generation, 8(2), 203-215.

Ringwood, J., Ferri, F., Ruehl, K., Yu, Y.-H., Coe, R., Bacelli, G., et al. (2017). A competition for WEC control systems. In 12th European wave and tidal energy conference, Cork.

Ringwood, J. V., Bacelli, G., \& Fusco, F. (2014). Control, forecasting and optimisation for wave energy conversion. IFAC Proceedings Volumes, 47(3), 7678-7689.

Ringwood, J. V., Bacelli, G., \& Fusco, F. (2014). Energy-maximizing control of wave-energy converters: The development of control system technology to optimize their operation. IEEE Control Systems, 34(5), 30-55.

Roessling, A., \& Ringwood, J. V. (2015). Finite order approximations to radiation forces for wave energy applications. Renewable energies offshore (pp. 359-366).

Rossiter, J., \& Kouvaritakis, B. (1993). Constrained stable generalised predictive control. In IEE proceedings d-control theory and applications: vol. 140 (pp. 243-254). IET.

Salter, S. (1979). Power conversion systems for ducks. In International conference on future energy concepts (pp. 100-108).

Schoen, M. P., Hals, J., \& Moan, T. (2011). Wave prediction and robust control of heaving wave energy devices for irregular waves. IEEE Transactions on Energy Conversion, 26(2), 627-638.

Scruggs, J. (2011). Multi-objective optimal causal control of an ocean wave energy converter in random waves. In Oceans 2011, Waikoloa (pp. 1-6). IEEE.

Scruggs, J., Lattanzio, S., Taflanidis, A., \& Cassidy, I. (2013). Optimal causal control of a wave energy converter in a random sea. Applied Ocean Research, 42, $1-15$.

Sira-Ramirez, H., \& Agrawal, S. K. (2004). Differentially flat systems. CRC Press.

Soltani, M. N., Sichani, M. T., \& Mirzaei, M. (2014). Model predictive control of buoy type wave energy converter. IFAC Proceedings Volumes, 47(3), 1115911164.

Tedeschi, E., Carraro, M., Molinas, M., \& Mattavelli, P. (2011). Effect of control strategies and power take-off efficiency on the power capture from sea waves. IEEE Transactions on Energy Conversion, 26(4), 1088-1098.

Tedeschi, E., Molinas, M., Carraro, M., \& Mattavelli, P. (2010). Analysis of power extraction from irregular waves by all-electric power take off. In Energy conversion congress and exposition, Atlanta (pp. 2370-2377). IEEE.

Teillant, B., Gilloteaux, J.-C., \& Ringwood, J. V. (2010). Optimal damping profile for a heaving buoy wave energy converter. IFAC Proceedings Volumes, 43(20), 360-365.

Thomas, G., \& Evans, D. (1981). Arrays of three-dimensional wave-energy absorbers. Journal of Fluid Mechanics, 108, 67-88.

Tom, N. (2014). Confirmation of a nonlinear model predictive control strategy applied to a permanent magnet linear generator for wave-energy conversion. In 33rd international conference on ocean, offshore and arctic engineering, San Francisco. American Society of Mechanical Engineers V09BT09A057.

Tom, N., \& Yeung, R. W. (2013). Performance enhancements and validations of a generic ocean-wave energy extractor. Journal of Offshore Mechanics and Arctic Engineering, 135(4), 041101.

Tom, N., \& Yeung, R. W. (2014). Nonlinear model predictive control applied to a generic ocean-wave energy extractor. Journal of Offshore Mechanics and Arctic Engineering, 136(4), 041901.

Tona, P., Nguyen, H.-N., Sabiron, G., \& Creff, Y. (2015). An efficiency-aware model predictive control strategy for a heaving buoy wave energy converter. In 11th European wave and tidal energy conference, Nantes. 
Venkat, A. N., Hiskens, I. A., Rawlings, J. B., \& Wright, S. J. (2008). Distributed MPC strategies with application to power system automatic generation control. IEEE Transactions on Control Systems Technology, 16(6), 1192-1206.

Vicente, P. C., Antonio, F. d. O., Gato, L. M., \& Justino, P. A. (2009). Hydrodynamics of multiple floating point-absorber wave energy systems with inter-body and bottom slack-mooring connections. In 28th international conference on ocean, offshore and arctic engineering, Honolulu (pp. 1217-1227). American Society of Mechanical Engineers.

Villa Jaén, A., García-Santana, A., \& Montoya-Andrade, D. E. (2014). Maximizing output power of linear generators for wave energy conversion. International Transactions on Electrical Energy Systems, 24(6), 875-890.

Von Stryk, O., \& Bulirsch, R. (1992). Direct and indirect methods for trajectory optimization. Annals of Operations Research, 37(1), 357-373.

Wächter, A., \& Biegler, L. T. (2006). On the implementation of an interior-point filter line-search algorithm for large-scale nonlinear programming. Mathematical Programming, 106(1), 25-57.
Wang, L. (2001). Continuous time model predictive control design using orthonormal functions. International Journal of Control, 74(16), 1588-1600.

Westphalen, J., Bacelli, G., Balitsky, P., \& Ringwood, J. V. (2011). Control strategies for arrays of wave energy devices. In 9th European wave and tidal energy conference, Southampton.

Williams, P. (2004). Application of pseudospectral methods for receding horizon control. Journal of Guidance, Control, and Dynamics, 27(2), 310-314.

Wright, S., \& Nocedal, J. (1999). Numerical optimization: vol.35. Springer Science.

Yemm, R., Pizer, D., \& Retzler, C. (2002). Floating apparatus and method for extracting power from sea waves. US Patent 6,476,511.

Yu, Y.-H., \& Li, Y. (2011). Preliminary results of a RANS simulation for a floating point absorber wave energy system under extreme wave conditions. National Renewable Energy Laboratory.

Zheng, A., \& Morari, M. (1994). Stability of model predictive control with soft constraints. In 33rd IEEE conference on decision and control, Florida. 